Illinois State University

ISU ReD: Research and eData

Theses and Dissertations

4-3-2020

\title{
Anxiety In Presentational And Interpersonal Oral Tasks Face-To- Face And Online
}

Angus Leydic

Illinois State University, leydicangus1@gmail.com

Follow this and additional works at: https://ir.library.illinoisstate.edu/etd

Part of the Instructional Media Design Commons, and the Linguistics Commons

\section{Recommended Citation}

Leydic, Angus, "Anxiety In Presentational And Interpersonal Oral Tasks Face-To-Face And Online" (2020). Theses and Dissertations. 1244.

https://ir.library.illinoisstate.edu/etd/1244

This Thesis is brought to you for free and open access by ISU ReD: Research and eData. It has been accepted for inclusion in Theses and Dissertations by an authorized administrator of ISU ReD: Research and eData. For more information, please contact ISUReD@ilstu.edu. 


\section{ANXIETY IN PRESENTATIONAL AND INTERPERSONAL ORAL TASKS FACE-TO- \\ FACE AND ONLINE}

\section{ANGUS LEYDIC}

\section{Pages}

Within computer mediated communication, there has been a large focus on synchronous and asynchronous text-based methods and how it can be used to help oral proficiency and lower anxiety but very few studies have looked at how audio computer mediated communicative tasks affect anxiety. This study sets out to seek whether using audio computer mediated communication helps lower anxiety for beginner Spanish language students. In order to do so, three research questions are suggested: (1) How do participants' levels of FLA differ between presentational oral tasks completed in the classroom and presentational tasks completed in an online interface? (2) How do participants' levels of FLA differ between interpersonal oral tasks completed in the classroom and interpersonal tasks completed in an online interface? (3) What factors are associated with different levels of FLA in oral tasks completed in the classroom and oral tasks completed in an online interface? To answer these questions, two first-semester first-year Spanish classes completed one presentational oral task in the classroom and online, one interpersonal oral task in the classroom and online and completed an anxiety survey as well as answered open-ended questions after each task in order to better understand their thought processes. The overall results showed that when looking at fear of negative evaluation and communication apprehension, the participants indicated lower anxiety when completing online tasks. However, when looking at general anxiety, participants reported higher levels of anxiety. 
KEYWORDS: Foreign Language Anxiety, Computer Mediated Communication, Educational Technology, Spanish 
ANXIETY IN PRESENTATIONAL AND INTERPERSONAL ORAL TASKS FACE-TO-

FACE AND ONLINE

ANGUS LEYDIC

A Thesis Submitted in Partial

Fulfillment of the Requirements

for the Degree of

MASTER OF ARTS

Department of Languages, Literatures and Cultures

ILLINOIS STATE UNIVERSITY

2020 
(C) 2020 Angus Leydic 
ANXIETY IN PRESENTATIONAL AND INTERPERSONAL ORAL TASKS FACE-TO-

FACE AND ONLINE

\author{
ANGUS LEYDIC
}

COMMITTEE MEMBERS:

Montserrat Mir, Chair

Rachel Shively

Susan Hildebrandt 


\section{ACKNOWLEDGMENTS}

I would like to acknowledge Dr. Montserrat Mir for allowing me to complete this thesis with her and work on professional development through this as I learned how research should be conducted in our field. I would also like to thank my other committee members, Dr. Rachel Shively and Dr. Susan Hildebrandt for being willing to devote their time and energy to this arduous project. Finally, I would like to acknowledge my family and friends that have helped me through my anxiety when writing about anxiety.

A.L. 


\section{CONTENTS}

Page

ACKNOWLEDGMENTS

$\begin{array}{ll}\text { CONTENTS ii } & \text { ii }\end{array}$

TABLES

FIGURES

CHAPTER I: INTRODUCTION 1

CHAPTER II: LITERATURE REVEIW

Anxiety 3

$\begin{array}{ll}\text { Foreign Language Anxiety } & 7\end{array}$

FLA and Cognition $\quad 9$

FLA and Achievement $\quad 11$

Measuring Foreign Language Anxiety in the Classroom 12

$\begin{array}{ll}\text { Communicative Language Teaching } & 17\end{array}$

$\begin{array}{ll}\text { Computer-Mediated-Communication } & 18\end{array}$

FLA in CMC Xontexts 22

Motivation for This Study and Research Questions 25

CHAPTER III: METHODOLOGY 27

$\begin{array}{ll}\text { Participants } & 27\end{array}$

$\begin{array}{ll}\text { Spanish Course Curricula } & 27\end{array}$

Instruments and Procedures 29

$\begin{array}{ll}\text { Data Analysis } & 32\end{array}$

CHAPTER IV: RESULTS 
Research question 1: How do participants' levels of FLA differ between presentational oral tasks completed in the classroom and presentational oral tasks completed in an online interface? Research question 2: How do participants' levels of FLA differ between interpersonal oral tasks completed in the classroom and interpersonal oral tasks completed in an online interface?

Research question 3: What factors are associated with different levels of FLA in oral tasks completed in the classroom and oral tasks completed in an online interface?

Peer Comparison

Test Anxiety

Communication Apprehension

Preparedness

Summary of Results

Research question 1: How do participants' levels of FLA differ between presentational oral tasks completed in the classroom and presentational oral tasks completed in an online interface?

Research question 2: How do participants' levels of FLA differ between interpersonal oral tasks completed in the classroom and interpersonal oral tasks completed in an online interface?

Research question 3: What factors are associated with different levels 
of FLA in oral tasks completed in the classroom and oral tasks completed in an online interface?

$\begin{array}{ll}\text { CHAPTER VI: CONCLUSION } & 68\end{array}$

$\begin{array}{ll}\text { REFERENCES } & 72\end{array}$

APPENDIX A: STUDENT BACKGROUND INFORMATION 83

APPENDIX B: IN-CLASS PRESENTATIONAL TASKS 84

APPENDIX C: ONLINE PRESENTATIONAL TASKS 85

APPENDIX D: IN-CLASS INTERPERSONAL TASKS 86

$\begin{array}{ll}\text { APPENDIX E: ONLINE INTERPEROSNAL TASKS } & 87\end{array}$ 


\section{TABLES}

Table

Page

1. Means of Survey Questions between Online and In-Class Tasks 


\section{FIGURES}

Figure

Page

1. Responses to Q1, I usually feel nervous during the performance of oral tasks in class.

2. Responses to Q2, I tremble when I need to speak in Spanish in front of other classmates.

3. Responses to Q3, I don't worry about making mistakes when I perform oral tasks in class.

4. Responses to Q4, Even though I complete many oral tasks during the class, I feel nervous about it in class.

5. Responses to Q5, When performing oral tasks in class, I can get so anxious that I forget things I know.

6. Responses to Q6, It worries me that my classmates seem to speak Spanish better than I do.

7. Responses to Q1, I usually feel nervous during the performance of oral tasks online.

8. Responses to Q2, I tremble when I need to speak in Spanish with my partner online.

9. Responses to Q4, Even though I complete many oral tasks during the class, I feel nervous about it online.

10. Responses to Q4, Even though I complete many oral tasks during the class, I feel nervous about it online.

11. Responses to Q5, When performing oral tasks online, I can get 
so anxious that I forget things I know.

12. Responses to Q6, It worries me that my speaking partner online seems to speak Spanish better than I do. 


\section{CHAPTER I: INTRODUCTION}

Language learning is a mandatory part of the curriculum for many universities which calls upon a need to understand how people learn a language and how to make the process easier for them. Foreign language anxiety (FLA), a type of anxiety associated with second language learning, is a common field of study when it comes to second language acquisition as it can affect many different parts of the language learning journey such as academically, socially and cognitively (Zheng 2008). Within the last two decades major research dealing with Computer Assisted Language Learning (CALL) and Computer Mediated Communication (CMC) have looked at how to use technology to improve language learning and within this, FLA has been a major focus (Baralt \& Gaffney, 2011; Cho \& Carey, 2001; Melchour-Couto, 2017, 2018; Poza, 2011).

Research in oral speech within CALL and CMC has mainly focused on the relationship between oral task types and anxiety (Arnold, 2007; Baralt \& Gurzynski, 2011; Côte and Gaffney, 2018; Warshauer, 1996), or the transference of skills between a text-based CMC and face-to-face oral tasks (Brooks 2009, Kirkgoz, 2011). Text-based, and more recently, audio-based tasks could be done either asynchronously or synchronously, however, little research exists that focuses on audio-based CMC, using audio conferencing software, (Cho \& Carey, 2001; Poza, 2011) or even video-based CMC (e.g. zoom) and anxiety, with the exception of some research (Melchour-Couto 2017, 2018) in virtual worlds (e.g. video games).

This thesis hopes to create more research on the interaction of audio-based CMC and anxiety and provide some insight on how the medium and type of task affect anxiety. In order to do this, the thesis is set up into a literature review that looks at a portion of the large volume of literature published on anxiety, FLA, CMC and the relationship between CMC and anxiety. The literature review section hopes to provide key definitions, how FLA is measured, the importance 
of CALL approaches and insight on what research is available and why this research on audio CMC is important and lacking. After this, the methodology section is set up to explain the context of the study, how the oral tasks were carried out, the means of data collection and how the data will be interpreted. All data in the research section is organized by research question and the use of quantitative data is explained by supporting qualitative data. At the end of the results chapter, there is a brief summary outlining key features of the data. The discussion chapter looks at interpreting the results obtained and explaining where this data fits among previous studies based in CMC and CALL approaches. The conclusion then summarizes all the given information, goes over pedagogical implications, explains the limitations of the study and offers suggestions for future research. 


\section{CHAPTER II: LITERATURE REVIEW}

The literature review is set out to give a background of relevant information that pertains to the study at hand and provide important definitions that are needed in order to complete an analysis of the data. First, we look at the history and definitions of anxiety, and FLA while examining its effects on foreign language learning. Next, a brief section on communicative language teaching is offered to contextualize the teaching setting for the current study. We also explore the fields of After CALL and CMC with a special focus on anxiety related research. We end this chapter with motivations for the present study and its research questions.

\section{Anxiety}

Anxiety reflects a feeling of discomfort with a situation that can be perceived as threatening (Koba et al., 2000). Anxiety is a mood that may be provoked without a physical stimulus, is unavoidable and has long duration (Ohman, 2000). Anxiety affects highly anxious individuals by diverting attention between relevant (e.g. problem-solving) and irrelevant thoughts (e.g. worrying) (Macher et al., 2013). Even with new studies coming out on anxiety, much research still relies the following seminal studies in order to understand and measure anxiety.

Within the realm of general anxiety, one large factor that plays a role in how nervous someone feels is communication apprehension, which is the fear or anxiety an individual feel about oral communication (Richmond, 1985). Since anxiety is emotion based, provoking the arousal of the limbic system plays an important part in communication (Lamendella, 1977). There are other constructs not directly tied to orality, but which nonetheless emphasize people's anxieties about communication such as writing apprehension, touch avoidance, and receiver apprehension (Daly, 1991). Anxiety can also be created by believing one will experience anxiety in a situation. Some examples would be a fear or an obsession of possible ways the speaker is able to ruin a potential 
situation or conversation in the future (Richmond, 1985). Others might believe they will speak incorrectly or answer wrong while completing different types of oral tasks which means the anxiety will continue through the whole task itself.

Anxiety has since been separated into state, trait and situational anxiety. Although no clear delineation between these three categories exists, Zheng (2008) declared "the differences can roughly be identified on a continuum from stability to transience, with trait anxiety related to a generally stable predisposition to be nervous in a wide range of situations on one end, and a moment-to-moment experience of transient emotional state on the other" (p. 2). Ellis (1994) defines state anxiety as "...the apprehension that is experienced at a particular moment in time as a response to a definite situation," (p. 693) which refers to a "transitory state or condition of the organism that varies in intensity and fluctuates over time" (Spielberger, 1966, p. 12).

Scovel (1978), on the other hand, defines trait anxiety as a personality trait referring to a "permanent predisposition to be anxious" (p. 479). Trait anxiety, however, is the way that an individual experiences anxiety without considering different situational differences such as a dreaded or threatening environment (Spielberger, 1972). This refers to individuals who feel generally anxious in situations which do not usually provoke anxiety. Trait-like communication apprehension is "a relatively enduring, personality-type orientation toward a given mode of communication across a wide variety of contexts" (McCroskey, 1984, p.16). This means that a person's anxiety score should be similar during an extended period of time without any type of intervention. Considering the difference between state and trait anxiety, performance from people who suffer from high levels of trait anxiety is generally lower than those who do not suffer from high trait anxiety (Horwitz, 2002). 
These two definitions, trait and state anxiety, left anxiety as a binary construct when it should be considered as a spectrum. Ellis (1994) defines situational anxiety as anxiety experienced that is context specific such as public speaking. Daly (1991) explains that within situational anxiety there are four types of anxiety that need to be looked at: (1) evaluation apprehension, (2) novelty apprehension, (3) ambiguity and (4) conspicuousness. Evaluation apprehension appears whenever there is a type of assessment for example, an interview for a job. Another type of situational anxiety is novelty apprehension which happens due to the familiarity of the situation and people involved. The less familiar the situation, the more apprehensive one may be. These situations include unfamiliar problems, and tasks. Problems in language research may also deal with novelty as speaking a new language is a different situation in which students find themselves and therefore, may influence their anxiety levels. Ambiguity is another factor that affects anxiety. Similarly to the idea of novelty, if the students do not know what they are being assessed on or what is going to happen, their anxiety levels might increase. Finally, conspicuousness affects anxiety when a student feels that they are the center of attention which may happen when one needs to answer questions in class.

Situational anxiety falls in the middle of the continuum between state and trait anxiety denoting the probability of becoming anxious in a particular type of situation. Students who experience situational anxiety may remember moments in which their anxiety was high and when they face a similar obstacle, past experiences may provoke communication apprehension, for example, fear of negative evaluation (Onwuegbuzi et al.,1991).

Communication apprehension has been defined as a type of state anxiety stimulated in certain environments as public speaking which can come from a fear of being negatively evaluated (Horwitz, 1991). Initially, communication apprehension was referred to a type of anxiety that 
happens when someone fears actual or anticipated communication with a person or a group of people impacting their social skills or self-esteem (Holbrook, 1987). Later, a more precise definition was proposed that described communication apprehension as stage fright, communication anxiety, or performance anxiety (Horwitz, 1991). In addition, Richmond (1985) has looked at communication apprehension as a construct with four different aspects which are found on a continuum from trait-like to situational communication apprehension as follows "(1) communication apprehension as a trait, (2) communication apprehension as a generalized context, (3) communication apprehension with a given audience across situations, and (4) communication apprehension with a given individual or group in a given situation" (p. 32).

Trait-like communication apprehension (\#1) is defined as being similar to a trait, such as eye color, but rather than being static, it comes from highly change-resistant personality variables such as extroversion and introversion (Richmond, 1985). Therefore, there is a rigidness to the apprehension and anxiety that is experienced when communication apprehension falls into this category. In defining context-based communication apprehension (\#2), Richmond (1985) explains it "relates to people who are fearful or anxious about communicating in one type of context, while having no fear or anxiety in other contexts" (p. 34) which is highly reflective of the anxiety experienced in public speaking, interviews, and meeting new people. Audience-based communication apprehension (\#3) focuses more on concerns of reactions to communication with a certain individual, individuals or groups of people, while with others, they may not experience any type of anxiousness. This type of apprehension is focused more on the situational constraints rather than personality type of the individual and it is more enduring than situational communication. Finally, situational communication apprehension (\#4), although similar to context-based communication apprehension in that they are both "a response to situational 
constraints generated by the other person or group" (McCroskey 1984, p. 19), it varies in that situational communication apprehension is focused on a given situation at a given time while context-based refers to a "single type of communication context cutting across receiver and time" (McCroskey 1984, p. 19).

According to Daly (1991), to reduce communication apprehension the first tactic is to develop the skill that is associated with the anxiety experienced (e.g. public speaking). However, some studies have found that taking classes to teach skills on how to control anxiety can actually adversely affect apprehension to communicate (Richmond, 1984; Richmond \& McCroskey, 1988). One behavioral reaction to anxiety is the desire to avoid situations which spike anxiety within students as individuals who feel anxious about communicating often avoid opportunities to enhance their communications skills (Daly, 1991). However, as students are forced into a language course that requires them to speak, apprehensive students or high anxiety students do not have a choice in which class they enter or how the class is taught (Horwitz, 2002).

\section{Foreign Language Anxiety}

Researchers have looked at anxiety and foreign language learning, but there is a fundamental difference between anxiety and FLA. One of the seminal works on FLA comes from Horwitz, Horwitz \& Cope (1986) who were among the first to define FLA and also created a measuring technique that is still used in many current studies today, as the current one described here. Horwitz et al. (1986) viewed language anxiety as "a distinct complex of self-perceptions, beliefs, feelings and behaviors related to classroom language learning arising from the uniqueness of the language learning process" (p. 128) which highlighted unique circumstance that arise from having to learn and communicate in a new language. 
According to Horwitz et al. (1986) FLA is composed of three elements: (1) communication apprehension, (2) test anxiety, and (3) fear of negative evaluation. Communication apprehension is "a type of shyness characterized by fear of or anxiety about communicating with people" (Horwitz et al., 1986, p. 127). For both spontaneous and presentation speech, the effects of anxiety are clear, "difficulty speaking in dyads or groups (oral communication anxiety) or in public (stage freight), or in listening to or learning a spoken message (receiver anxiety) are all manifestations of communication apprehension" (Horwitz et al., 1986, p. 127).

Communication apprehension is especially important in foreign language learning because at high levels of anxiety, students may become forgetful, fearful of misspeaking, experience worry or even dread, and may exhibit physical symptoms as well such as sweating and palpitations (Horwitz et al., 1986). One of the biggest challenges in a foreign language classroom is engaging students in communication practice and considering that the majority of classrooms are now communicative based, this means that students with anxiety may suffer even more. For example, some students tend to forget words or freeze up when they need to practice spontaneous speech but can easily perform rote-drill exercises and presentation speech without much difficulty (Horwitz et. al., 1986).

The second type of FLA is test anxiety which "refers to a type of performance anxiety stemming from a fear of failure" (Horwitz et al., 1986, p. 127). Oral tests have the potential of provoking both test and oral communication anxiety simultaneously in susceptible students.

Finally, fear of negative evaluation is defined as "apprehension about other's evaluations, avoidance of evaluative situations, and the expectation that others would evaluate oneself negatively" (Horwitz et. al., 1986, p.128). This type of anxiety differs from test-anxiety as it encompasses a larger scope, further than just making mistakes on tests, but including any situation 
in which there may be an evaluation such as an interview or speaking in class. Additionally, fear of negative evaluation derives not only from students' peers but also from the instructor.

Communication apprehension, test-anxiety and fear of negative evaluation are all interrelated within a communicative based classroom in which students constantly have to produce oral language. In a typical class, students have to communicate with each other and their professor, risking negative evaluation by their peers and instructor. If they are assessed that day, they also have the addition of test-anxiety that may inhibit their cognitive abilities when processing input and producing output either orally or in written communication. Therefore, these three different anxieties may play a role in a student's ability to perform within one sole task.

Onwuegbuzie et al. (1999) correlated FLA with other individual learner variables to see what factors can predict FLA. He found that self-worth and self-esteem are significantly related to anxiety, which should not come as a surprise since fear of negative evaluation also affects social settings in which judgement of an interlocutor may spike anxiety due to the anxious student's perceived perception of their conversation partner. This means that if a speaker feels that the receiver may judge the speaker for their message, that can cause anxiety.

\section{FLA and Cognition}

With regard to mental functioning, Dörnyei (2009) describes cognition as a construct "associated with knowing and knowledge representation, memory, attention, learning, information processing, abstract thinking, appraisal, judging, reasoning, problem-solving, decision-making, etc.” (p. 202). Cognition can be hard to explain and capture in research as it cannot be characterized as a linear computational sequence between input and output functions but rather as a dynamic process of self-organization with only partial predictability from moment to moment (Lewis, 2005). Anxiety arousal is associated with cognitive disruptions and distractions often associated 
with self-focused thinking (Norton \& Abbott, 2016). Since anxiety is so closely connected with cognition, it "is quite possibly the affective factor that most pervasively obstructs the learning process" (Arnold \& Brown, 1999, p. 8).

One negative affect of language anxiety is its interference with cognitive processing at the input, processing (incorporation of new info into long-term memory) and output stages (MacIntyre \& Gardner, 1994a; Tobias, 1986; Onwuegbuzie et al., 2000). During input, anxiety may cause attention deficits and poor initial processing of information. In short, not as much information is registered. Vogely (1998) found that FLA can affect the need to process input rapidly, such as in spontaneous oral tasks. People with higher anxiety seem easily distracted from the task because time is divided between the processing of emotional-related and task-related cognition while at the processing stage, if the task is relatively simple, anxiety may have no effect (MacIntyre \& Gardner, 1989). However, as the task becomes more difficult, relative to ability, anxiety shows greater impact on processing. Interference with the rehearsal of new information would be an example of this type of effect. At the output stage, anxiety may interfere with the retrieval of previously learned information. MacIntyre (1995) also believed that FLA forms a part of social anxiety which derives from the social and communicative aspects of language learning, such as the need to interact as a group and use the target language to complete tasks. In his article, he defends Tobias' (1986) view on cognition and anxiety as well as other individual learner variables.

Process-based work models have allowed researchers in second language acquisition make predictions about performance. Levelt's (1989) model understands that language storage in the short-term memory has importance in second language acquisition and production. The Working Memory theory allows us to model how the brain collects and maintains temporary visual and visual-spatial information which is used for executing judgement and functions (Payne \& Whitney, 
2002). This process reflects the cognitive process of oral exchanges as students have to take in input, processes the received information, make judgements about what was said and then create the appropriate output. Dewaele (2002) concluded that high anxiety, especially when linked with high introversion, can lead to breakdowns in automatic processing and therefore can seriously hinder second language (L2) fluency because L2 use depends on the effectiveness of the employment to attentional resources and of the working memory in particular.

\section{FLA and Achievement}

The relationship between FLA and achievement has been widely researched. Some early studies reported a negative relationship between anxiety and achievement in foreign language learning meaning that the higher the anxiety, the lower the scores students would receive, or vice versa (Clément et.al.,1977, 1980). However, other studies claimed a positive or no correlation at all between these two factors which caused for a need in understanding and conceptualizing FLA (Kleinmann, 1977; Scovel, 1978).

More recently however, most second language acquisition literature demonstrates that high levels of language anxiety are associated with lower levels of language achievement (Dewaele, 2007; Gardener \& MacIntyre, 1993; Horwitz, et al., 1986; MacIntyre \& Gardner, 1991a, b, c; Onwuegbuzie et al., 1999, 2000, 2002; Sanchez-Herrero \& Sanchez, 1992; Woodrow, 2006). For example, MacIntyre (1999) says that FLA is one of the biggest predictors for academic achievement which reflects the importance of anxiety within the FL classroom while Onwuegbuzi et al. (1999) found that language anxiety is one of the best predictors for language proficiency.

Horwitz et al. (1986) pointed out that anxiety blocks second language acquisition and learning. In fact, it has been shown that strong anxiety feelings are disruptive to behavior, interfering with interpersonal communication, cognition, and learning (MacIntyre \& Gardner, 
1994). For example, students with higher levels of anxiety receive lower course grades and are more likely to want to drop out of their language course (Dewaele, 2009). However, mild levels of self-reported anxiety as reported in Dewaele et al., (2016) may not affect results as significantly as high levels of anxiety. Interestingly enough, anxiety may also be related to other personality traits like perfectionism (Gregersen \& Horwitz, 2002). Therefore, some students who experience high levels of anxiety and low academic achievement may spend more time studying or 'over studying' (Horwitz et al., 1986).

A more current topic of study in anxiety research is the relationship between enjoyment and anxiety. Boudreau et al. (2018) found a clear negative correlation between anxiety and enjoyment when it comes to language learning although a few participants in the study deviated from this result. For example, one participant reported similar levels of anxiety and enjoyment and attributed his enjoyment to liking learning French. Therefore, some people may experience anxiety but also enjoyment under the same situation which suggests that enjoyment does not necessarily reduce anxiety for language learners. A similar conclusion was reached by Dewaele et. al. (2016) who also found that even though higher levels of enjoyment are related with successful learners, there is still anxiety within those learners.

\section{Measuring Foreign Language Anxiety in the Classroom}

Historically, studies (Swain \& Burnaby, 1972; Tucker et al., 1976) have shown incomplete correlations between anxiety and measures of language proficiency. Anxiety may come from different sources which has caused problems understanding FLA in beginner language learners and advanced language learners. In addition, some studies have found a constant correlation between academic performance and an anxiety measure including in measures of FLA (Backman, 1976; Chastain, 1975). However, other studies found inconsistencies in this correlation (Scovel, 
1978). Scovel's research (1978) on anxiety on foreign language and second language learning found mixed and confusing results; this also happened with Chastain's (1975) study that reported a mix of positive, negative and near zero correlation between anxiety and second language learners. These discrepancies in these early studies come from a lack of a unified understanding of what FLA is and how to measure it properly.

In one of the first reviews of anxiety and language learning, we also see inconsistent results across 16 studies examined (Young,1991). However, in MacIntyre's (1999) examination of the same studies he claimed that the inconsistency was because the anxiety monitored in foreign language studies was not specific to the second language acquisition context. MacIntyre asserts that previous research from Clément et al., (1977, 1980), Symthe, Clément and Gliksman (1976), and Gardner et al., (1984) all used surveys and figures that reflected general anxiety, and attitude and motivation. For this reason, early studies on FLA are often overlooked by other researchers due to their inconsistent correlations. Gardner et al. (1979) was one of the first studies that measured anxiety while looking at it as a construct unique to foreign language learning as they researched FLA in French as a second language students. These early studies on FLA focused mostly on academic achievement and ignored other aspects that anxiety may affect such as performance.

Differences in measurement that led to many inconsistencies in anxiety research motivated Horwitz et al., (1986) to create the Foreign Language Classroom Anxiety Survey (FLCAS). Horwitz et al. (1986) looked at a support group focusing on issues in foreign language learning among students at the University of Texas during the summer of 1983 and noticed a pattern in the way participants spoke about their reactions to speaking and learning a new language. Inspired by these patterns, the researchers set out to define FLA as a separate form of anxiety. By having 
students complete a series of anxiety scales at different stages of classroom tasks completion, it was found that the anxiety experienced in the foreign language classroom is different than the normal definition of anxiety which led to the need for a clear scale of their own to measure this new type of anxiousness (Horwitz et. al., 1986).

In Horwitz et al.’s (1986) study, they conceptualized FLA along with a 33-item FLCAS questionnaire. This questionnaire revolutionized language anxiety research as it separated language anxiety from general anxiety giving more cohesive and less erratic results. The study determined that language anxiety is related to apprehension about communicating, fear of negative evaluation by others and test anxiety. Drawing from Horwitz et al.'s (1986) results on FLA, MacIntyre (1999) defines language anxiety as the "worry and negative emotional reaction aroused when learning or using a second language" (p. 27). Today language anxiety is understood as an independent factor, meaning that this is not merely a transfer of test anxiety or communication apprehension, but it is a uniquely L2-related variable (Horwitz, 2001; MacIntyre,1999, 2001, 2002; MacIntyre \& Gardner, 1991a, 1991b, 1994).

The original FLAC survey developed by Horwitz et al. (1986) has been used in many studies since its creation but recently, Park (2014) pointed out that the scale does not classify FLCAS items into the three different elements of FLA as communication apprehension, fear of negative evaluation and test anxiety but rather were suggested as analogies for FLA (Horwitz 2016) . As each item is not defined into the three types of anxieties (communication apprehension, fear of negative evaluation and test anxiety) on the FLCAS, there may be misinterpretations of the three components in the measurements. Park (2014) also speculates that with studies that use students who do not speak English as their native language, there can be inconsistencies in the translation of the scale which can result in unreliability of the results. The issue with translation 
may result in differences in factor analysis and variation for responses in FLCAS (Park 2014). Horwitz (2016) also mentions that cultural background can also cause increases of FLA due to customs with how speech is looked at and how important negative evaluation is to different cultures.

Horwitz 's original FLCAS scale (1986) reflects the orality of language and might not be suitable for realistic measures of anxiety with writing and reading. To this day, researchers still do not understand if FLA is just one type of anxiety or if it is an ensemble of different types of anxieties, such as listening anxiety, reading anxiety, writing anxiety etc. For example, Elkhafaifi (2005) suggests that FLA and listening anxiety are separate but related. He found in his study on Arabic language students that although these two types of anxiety are distinct, they both affect foreign language achievement. Since FLCAS mainly relates to speaking in the foreign language, a majority of the literature on FLA has focused on speaking (Horwitz \& Young, 1991; Saito et al.,1999), but within the last two decades more studies have investigated other skills such as reading (Young, 1992), writing (Cheng, 2002), speaking (Young, 1992) and listening (Elkhafaifi, 2005; Vogely, 1998).

Furthermore, FLCAS was created in the context of English-speaking students learning French, which means that the socio-dynamics of anxiety and culture were not explored in its creation as it only took one culture into consideration. Therefore, studies that use students from other cultures such as Asian cultures, would have to be cognizant of how cultural differences, such as the importance of achievement, may affect anxiety (Park, 2014). As with cultural interference, other variables may affect how FLA is experienced, as is the case with gender. Some studies report that women have lower FLA than men (Campbell \& Shaw, 1994; Dewaele, et al., 2016; Kitano ,2001) others have concluded opposite findings (Arnaiz \& Guillén, 2012; Donovan \& MacIntyre, 
2005), and finally, some studies have found no difference in FLA manifestation between the two genders (Dawaele, et al., 2008). For example, one study shows that women reported that they felt "significantly more worried than their male peers. They tend to feel more nervous and confused $[\ldots]$ and felt that others were better at speaking the FL than they were" (Dewaele et al., 2016, p. $52)$.

The issue of how to measure FLA has also been explored. As mentioned, the FLACS is still been used to collect numeric data but to have a complete understanding of anxiety, qualitative and quantitative data are needed since self-reporting does not give researchers enough data to work out how to minimize anxiety (MacIntyre, 2012). Horwitz (2016) also suggests that reasons for FLA may vary on proficiency as a beginner language learner might worry about one pronunciation while advanced language learners might worry about conveying a message more.

For example, in Price's (1991) work, students were interviewed to described how they felt in their language class and what role anxiety played in their studies. Their responses helped researchers better understand FLA by moving away from quantitative data and giving more specific details and allowing for more focused recommendations on how to reduce FLA. More recently, Yan \& Horwitz (2008) sought to view how learner's perception of anxiety affected other learner differences through interviews. They found that students believe that FLA exerts influence over achievement and interests and motivation while learning strategies, comparisons with peers and interests and motivation all negatively affect FLA. Gregersen (2003) looked at frequency of errors made by anxious and non-anxious students through quantitative data but then to further explain his results, he sought to contextualize the numerical data with descriptions of participant comments. More and more researchers are trying to triangulate data using both qualitative and quantitative measures to try to better answer their research questions. 


\section{Communicative Language Teaching}

Brandl (2008) describes Communicative Language Teaching (CLT) as an approach that encompasses a variety of methods and approaches to assert that the focus of language is communication. CLT "changes the acquisition pattern of fundamental syntactic structures of students and emphasizes on the communicative function of language rather than grammar and vocabulary" (Wang \& Zhu, 2020). In summation, it facilitates language learning through real-life activities in order to teach all facets of language based on communicative competence. According to Brandl (2008), communicative competence is based on (1) linguistic competence, saying the appropriate thing at the appropriate time, (2) sociolinguistic competences, being able to carry out a full conversation in a coherent manner, (3) discourse competence, being able to communicate effectively, (4) strategic competency, being able to repair any miscommunications.

This type of teaching style moves past typical grammar drills and uses more tasks and projects in which students strive to work through challenges together in order to negotiate meaning allowing the students to create their own methods of having a conversation and working through any communication block and create their own repairs. Central to class work, activities and tasks must be relevant to a learner's needs, be motivating but challenging, elicit interaction, and involve communicative language use and metalinguistic reflection. Tasks are "real-world activities people think of when planning, conducting or recalling their day" (Long, 2015, p. 5). Through tasks, students are able to develop proficiency in the target language.

As CLT focuses on teaching language through communication in the classroom and there is a substantial time needed to learn a language, there became a need to have students work outside of the classroom on language learning. To answer this, Computer Assisted Language Learning 
increased in popularity as it allowed students to complete input-based tasks and drills before and after coming to class.

\section{Computer-Mediated-Communications}

Originally, Computer Assisted Language Learning (CALL), opened the doors to integrate educational technologies in language learning by the incorporation of drills and input-based tasks through software programs and internet-based programs such as learning management systems. CALL is "the search for and study of applications of the computer in language teaching and learning" (Levy, 1997, p.1). Through the use of technology, we can increase the scope of target language input, which will allow a lesser need of focus on input drills in the classroom setting (Chapelle, 2009). As technology has evolved, we are now able to focus on oral communication in the classroom and even extend oral target language use outside of the classroom with the use of text-based and audio based applications, and even newer technologies such as skype, facetime, and other mobile or computer applications.

CALL methods have now evolved into what researchers call Computer Mediated Communication (CMC), "real-time, synchronous conversation that takes place $[\ldots]$ via the internet' (Baralt \& Gurzynski-Weiss, 2011, p. 206). CMC used the internet to allow students to engage in language through different types of modalities (visual, audio and textual) (Hampel, 2006). With e-learning, researchers and instructors are trying to find new ways to integrate technology in the classroom and outside to facilitate learning. Some modes of communication,

such as social media, can increase students' contact and usage of the target language, although some claim that students do not want to use social media for learning (Ward et al., 2009). As such, most CMC studies rely on chatrooms, audio calls and video conferencing tools although the majority of students in the past have mostly focused on text-based CMC. 
Although Baralt \& Gurzynski-Weiss (2011) specifically defined CMC as synchronous, students can use CMC through either asynchronous or synchronous means. Asynchronous CMC is when two interlocutors use forums, emails or any other means of communication that are not instantaneous and they have time to think and work through their responses. Synchronous CMC is when a conversation takes place with all parties in the conversation present and communicating at the same time. Both asynchronous and synchronous CMC can take place in either text-based, audio-based or video-based chats.

CMC-based tasks can provide support for students that they might not feel that they get in class oral tasks. Abrams (2003) investigated the impact of online tasks on the amount of language produced. Participants were separated in three different groups to complete several oral tasks: control (face-to-face), synchronous CMC and asynchronous CMC. She saw that students who participated in the synchronous CMC produced more language than those in asynchronous and face-to-face chat groups. One reason for students to produce less in the asynchronous group is that sometimes students might not have been motivated or taken the task seriously and had to wait up to a week to receive a response from their partner. Abrams (2003) found that output production increased online, but students did not produce higher quality language. She measured this by lexical richness, diversity and syntactic complexity. She indicates that complexity is related to discourse cohesion and students who are able to join previously stated ideas or even scaffold their answers using their partners ideas are considered more complex. Abrams' (2003) findings called for alternate methods of analyzing language use and complexity. Conversely, Absalom and Pais Marden (2004) in their study on email conversations found that the language produced online is often more complex and/or more grammatically accurate than that produced in face-to-face interaction. The increase in complexity may be attributed to how in text-based CMC, online 
communication removes social barriers and provides greater equity of participation compared to classroom contexts and student autonomy and responsibility for learning increases (Absalom \& Pais Marden, 2004).

CMC methods vary in the support that they offer students. With asynchronous text-based CMC tasks, it is important to note that students have ample opportunity to both compose and revise their responses, with the assistance of other resources including more proficient language users. Côte and Gaffney (2018) state that another difference between synchronous CMC text chats and face-to-face oral tasks is that students can type at the same time and answer each other's questions as they keep sending messages. Both, the less urgency of a quick response and the lack of structured conversation, may be a factor as to why students interact more with each other through the text-based chat.

Studies in the CMC context have also looked at how blended classrooms and distance learning compare to the traditional classroom. Blake et al., (2008) looked at students in a university course which was offered in three different mediums - a traditional class, blended class — both online and face-to-face — and as a class offered to distance students through online means only. They looked at the use of a bimodal, both audio and text, chat to promote oral proficiency among first- and second-year Spanish students. They found that students in both the blended and the distance learning classes performed similarly to those in the traditional classroom. These results were in line with showing how text-based chats can help promote oral proficiency as well as how synchronous audio chats also promote the same growth as face-to-face oral practice.

Another line of research with CMC tasks has focused on student performance. Brooks' (2009) study on student performance in oral tasks in a CMC environment with an examiner and with student-formed dyads showed that students performed better when they were performing with 
another classmate. This is not to be mistaken with higher achievement, but rather participants had richer performances. Students who were put together interacted more, such as prompting explanations and repeating received information, and produced more linguistically complex forms. Brook's (2009) found that in testing scenarios student-student interactions appear to be more symmetrical than student-tester interactions meaning that the students provide each other ample opportunity to negotiate meaning and co-create conversation together. Similarly, Taylor (2000) found that in comparison to student-tester interactions, student-student dyadic paired work results in performance equivalently found in classroom paired work. It appears that the type of interaction in student-student conversations through $\mathrm{CMC}$ is quite similar to the types of interactions performed in a traditional classroom setting.

Finally, CMC research has also looked at video-conferencing and language learning. Kirkgoz (2011) evaluated the use of video-conferencing to complete oral tasks outside of the classroom. Students were set up into small groups and given different situations to complete. The students reported that their collaboration and co-creation of meaning allowed them to interact better with the language than in previous courses. They found that students were more wellprepared for tasks when they knew they would be recorded and that students typically enjoyed the video feature to self-assess their performance. They also concluded that students were producing more linguistically complex and more meaningful sentences after completing the videos a few times.

Besides gains in performance and linguistic complexity, CMC studies have also shown other interesting positive results. For example, web-based instructional activities benefit the performance of lower achieving students (Yu, et al., 2010) and result in increased motivation and 
self-concept, easier access and increased interaction between students and student-teacher interaction, and improved acquisition of basic skills (De los Arcos, et al., 2009).

\section{FLA in CMC Contexts}

Within CALL research, many studies have been carried out to see how text-based CMC can help students overcome anxiety (Arnold, 2007;; Baralt \& Gurzynski, 2011; Côte and Gaffney, 2018; Warshauer 1996), but very few have looked at anxiety and oral CMC, either audio or video. Research (Warshauer 1996) has implied that asynchronous and synchronous CMC can help reduce anxiety in foreign language students and even that students who do not participate orally in class discussions and tasks can benefit from the online format with little to no anxiety and stress.

Much of the research has focused on comparing language learning experience in face-toface and CMC settings. Arnold (2007) examined groups of language learners who participated in communicative tasks in face-to-face settings, in synchronous text-based chats and in asynchronous text-based chats using FLCAS. Participants only performed these tasks in one mode of communication and authors found that synchronous text-based chats were able to help lower students' anxiety just like with asynchronous text-based chats. One aspect of this lower anxiety is the anonymity of a CMC in which the student does not feel subjected to fear of negative evaluation. Warshauer (1996) explains that since students are working through a computer, they are not exposed to any paralinguistic input such as body language and they are not exposed to any age, race, or gender clues. These factors can help relax the student as they work through a synchronous or asynchronous text-based chat. Another aspect that helps lower anxiety in these situations is the time that the students are allowed to spend on working through the language. More so with asynchronous chats, the students are able to take their time to thoughtfully write out their answers instead of being forced to engage spontaneously and with little preparation. It can be speculated 
that in audio chats, either synchronous or asynchronous, these benefits would be transferable due to the anonymity of not seeing the other person whereas these benefits might not be applicable in video CMC as students would see each other.

In another study, Baralt and Gurzynski-Weiss (2011) who found that students expressed both positive and negative opinions about both face-to-face oral tasks and text-based CMC tasks. One student expressed their frustration about face-to-face oral communication as it is more obvious when you make a mistake which relates to a fear of negative evaluation from others. Others found CMC hard to deal with due to lack of visual cues making negotiation of meaning a little harder since it has to come from creating language rather than additional paralinguistic help such as body language and miming. These attitudes relate to anxiety experienced in the foreign language classroom as students can become frustrated and annoyed with tasks and their partner.

However, overall, participants in Baralt \& Gurzynski-Weiss'study (2011) reported similar anxiety levels in CMC tasks and face-to-face oral tasks. The authors reason that students are already familiar with using technology at home but having to interact with computers using a target language can cause a spike in anxiety. Students also spend almost double the time completing the CMC tasks than the face-to-face oral tasks which gives them time to double check their answers even if they are completing a synchronous task. In face-to-face communication students need to offer a quick response with little monitoring of the produced language. Students describe both face-to face and CMC tasks, as frustrating, anxious and stressful whereas CMC tasks are also described as confident (Baralt \& Gurzynski-Weiss, 2011).

Contrary to these studies that did not find relevant differences in FLA between face-to-face oral tasks and synchronous CMC text-based chats, Côte and Gaffney (2018) found lower anxiety in synchronous CMC text-based chats as compared to face-to-face oral tasks in class. Students 
who produce very little in classes might find comfort in participating in these types of written tasks in $\mathrm{CMC}$ rather than oral tasks. Since students need to produce output in order to better acquire a L2 (Swain 1985), this increased participation in forced output thought CMC text-based chats helps students gain more proficiency in the foreign language and reduce the variable of anxiety's effect on performance and proficiency. For example, Kern (1995) discovered that students using synchronous $\mathrm{CMC}$ text chats utilized more words during their task and took more turns using the language as compared to face-to-face communication. This difference could be due to the fact that students have more time to process input and plan output before answering.

More recently, interest in oral CMC has resulted in some exciting research. Oral CMC and its impact on anxiety levels has been studied in blended classrooms that use technologies that allow students to complete oral exercises online. Scida and Jones (2016) investigated a blended classroom for beginning Spanish university students and saw that based on a pre- and post-test FLCAS, anxiety in students dropped by the end of the semester with a large difference from the beginning to the end of the semester specifically in relation to communication anxiety. As with other studies (Cho \& Carey, 2001; Côte \& Gaffney, 2018; Poza, 2011), they found that the use of CMC helped lower anxiety in online learning.

Research on asynchronous voice computer conferencing in second language acquisition has revealed that the incorporation of online technologies on oral tasks such as the application Wimba could help reduce anxiety in students (Cho \& Carey, 2001; Poza, 2011). Poza (2011) also found that although students were anxious both in the classroom and in online setting, many students had lower anxiety when communicating through the asynchronous oral chats because they were less anxious about error correction from their professor. The results from FLCAS showed that test classroom anxiety was significantly higher than $\mathrm{CMC}$ anxiety which is congruent to 
Warshauer's (1996) ideas on how asynchronous CMC should work (i.e. lowering anxiety). However, one limitation of Warshauer's study (1996) was that the oral tasks completed in the classroom were synchronous whereas Wimba, in Poza's study (2011) is an asynchronous audio thread application which means that the anxiety experienced in both cases had other variables attached. As mentioned by Baralt and Gurzynski-Weiss (2011), asynchronous chats, whether textbased or oral, do not have the same urgency that classroom tasks inherently have which explains why the type and level of anxiety experienced in synchronous and asynchronous oral tasks may not be comparable.

Finally, other research has looked at the effects of using virtual worlds, such as video games, on anxiety when completing oral tasks. This is a type of synchronous audio CMC that allows students to participate within a certain level of anonymity. Melchor-Couto (2017) measured anxiety experienced in a traditional classroom and compared it to anxiety experienced in oral tasks completed in Second Life, a virtual world simulator. Second Life is a three-dimensional virtual world where users can pretend to be whomever they want by choosing an Avatar that represents their own identity. Within the two-hour classes, students completed four open-ended oral activities and the FLCAS and answered a few open-ended questions about their anxiety. Authors observed that anxiety levels were lower in students who participated in the online class and attributed the lower anxiety partly to the anonymity of Second Life. However, Melchor-Couto (2018) looked at audio CMC in Second Life again and found that there was no correlation between anonymity and anxiety. They also concluded that anxiety could be exacerbated by the lack of visual cues such as body language in students who need that type of paralinguistic support in order to negotiate meaning among students. 


\section{Motivation for This Study and Research Questions}

As discussed above, FLA is a pervasive affective variable that can affect language learning in all stages of cognition and through different mediums. Recent research involving technology seeks to discover whether CALL and CMC approaches can help students reduce FLA and promote better oral skills. However, the research is very limited which is the motivation for the current study. In many foreign language classes today, the limited amount of classroom time as well as the complex academic curriculum that needs to be covered, especially at the College level, limit the allotted time to communication in the classroom. As new technologies evolve, more foreign language classes are turning to these new technologies to enhance communication outside the classroom. There is, therefore, a need to explore whether oral CMC tasks impact anxiety levels the same way as face-to-face interaction in the classroom. In addition, it is also important to examine if different types of oral CMC tasks also affect students' anxiety levels differently. With this in mind, the current study sets out to investigate the following:

1. How do participants' levels of FLA differ between presentational oral tasks completed in the classroom and presentational tasks completed in an online interface?

2. How do participants' levels of FLA differ between interpersonal oral tasks completed in the classroom and interpersonal tasks completed in an online interface?

3. What factors are associated with different levels of FLA in oral tasks completed in the classroom and oral tasks completed in an online interface? 


\section{CHAPTER III: METHODOLOGY}

\section{Participants}

The data for this study was collected in one semester at a public university in central Illinois. Two first-year first-semester Spanish classes took part in this study with the final number of participants being 22 students; however, not every student completed each survey because of absences on the day data was collected. Therefore, a total of six participants completed all the activities. The researcher was the instructor in one of the courses and a TA was the instructor of the other course. All instructors teaching the same level, SPA 111, follow an established teaching schedule so both classes used for this study were very comparable. Student participation was voluntary, and all participants were recruited in the classroom using the appropriate IRB protocol and thus, all participants signed a consent form before the data collection took place. The participant pool was a mixed class of men and women with no native speakers nor heritage language speakers. Out of the 22 participating students, average age of the students was twenty years old.

Students at the beginning of the course filled out a background survey stating their name, age, year in college, interests, previous language experience, and exposure to the target language outside of the classroom in order to identify if there were any students who had a Spanish language background. Information from the background survey is used by teachers to create dynamic and authentic tasks relevant to the learner's interests, such as favorite TV shows, movies, books, etc. (Appendix A).

\section{Spanish Course Curricula}

The course used in this study, SPA111, is a first-year first-semester required course for students at Illinois State University. The course is designed for students with no prior background 
in Spanish. The objectives of the course are to offer a basic introduction to Spanish language and culture. Learning outcomes include how to greet each other, describe people, places and things, talk about likes and dislikes, discuss and describe family, talk about daily routines, and discuss university life. The students meet four times a week for a 50-minute class period, Monday through Thursdays. The curriculum follows a communicative approach using a Pearson's Arriba (ZayasBazán, 2005) textbook which focuses on communication. Each class hour is set up to complete different oral tasks while students complete more input-based and rote memory activities at home on the online platform, Pearson's MySpanishLab. On certain Saturdays, students complete an oral activity online either alone or with a partner, but for the purpose of this study, the classes participating in the study completed all the online oral activities in a language lab setting during a class period. On Sundays, the students had to complete a set of online activities based on what they learned in the previous week.

The curriculum for this introductory Spanish course covers the first five chapters of the Arriba book. For this course, students were tested with an online test after each chapter. At the end of the semester, there was a cumulative, in-class traditional final exam. In order to meet the course objectives, participants were required to do pre- and post-class preparation, to attend class regularly, and to participate fully in class activities. Prior to coming to class, students were asked to read and/or watch online video tutorials that present the vocabulary and grammar to be practiced in class the following day. In class, assigned content for the day was reviewed and put into practice extensively through activities with peers.

In class, students were encouraged to use the target language as much as possible as the instructor used Spanish approximately $90 \%$ of the class period. Many of class activities were guided practice leading towards more independent language in a more independent task. The final 
task in the lesson could be either interpersonal or presentational depending on the outcome of the day. All the previous activities completed reflect the form of the final task. If the final task is interpersonal, the activities completed were focused on conversation and group discussion while if the final task was presentational, the previous activities focused on interpreting presented information and presenting information rather than a discussion.

\section{Instruments and Procedure}

Participants in this study completed four oral tasks, two presentational and two interpersonal tasks. One of each type of task was performed in the classroom setting while the other was performed through the Pearson's MySpanishLab learning management system in a language lab setting. The online oral tasks were graded as dictated by the curriculum while the inclass oral tasks were not. Immediately after finishing the oral tasks, participants completed the anxiety survey which was administered online via Qualtrics Software Survey.

The Likert-scale anxiety survey (Appendix A) was adapted from Pyun (2013) which looked at affective variables in relationship to student task-based language learning attitudes. The original survey looked at the six questions that the researcher modified from Horwitz et al.'s (1986) FLCAS in order to fit a task-based teaching classroom. In addition, we added four open-ended questions to the survey to allow participants to explain their attitude during the oral tasks. The

questions were: (1) "How do you feel when completing the oral task? Why do you think you feel this way?" (2) Do your feelings change as you perform the oral task online?" (3) "How do you feel that the instructor could have helped you feel better before beginning the oral task online?" and 
(4) "How do you feel that you could have helped yourself feel better before entering the oral task online?

The first oral task they were asked to complete was a presentational oral task (Appendix B) describing their family tree. During this class period students participated in a 45-minute oral proficiency-based class which focused on family vocabulary through presentational activities. At the end of the class, the students were asked to describe their family tree in groups of four or five students. Each student spoke for 2 minutes approximately to describe their family tree to their group mates.

The second presentational oral task (Appendix C) was completed online through the learning management system, MySpanishLab. As mentioned, online oral tasks are usually assigned to be completed at home but for the purpose of this study, this oral task was completed in the language lab at the end of the lesson. The textbook for this Spanish course can be accessed online so the majority of students come to class with their own computer or tablet. However, to make sure everyone would have access to a computer, the decision was made to meet at the computer lab the days the online tasks were assigned for this study. Prior to this second presentational oral task, students had been learning how to conjugate and use reflexive verbs. In this lesson, learners completed four activities during a thirty-minute class period that reviewed using reflexive verbs and daily routine words. At the end of the class period, the students were asked to record a presentational task that asked them to describe their daily routines. The online activity was timed to eight minutes to complete but the recording was supposed to only last between 1 and 2 minutes. 
Both of these oral tasks were presented during classroom time but in different lessons although their implementation was quite similar. In both classes the students practiced using presentational skills and the vocabulary and grammar features needed to complete these tasks.

The in-class interpersonal oral task was completed after a forty-five-minute lesson on conjugating verbs. The students participated in mostly interpersonal tasks during the classroom period to prepare them for the final activity, an information gap activity on planning a party with their friends (Appendix D). Students had two columns of information in which they had to ask questions in order to get the answers. The activity was modeled in front of the students by the instructor, and the instructor encouraged only the use of the target language during the whole class period. The in-class interpersonal task lasted 10 minutes.

The online interpersonal oral task took place during a lesson where students completed a few review activities on daily routine and reflexive verbs which focused on interpersonal communication between dyads. The students then completed an information-gap activity where information had to be gathered from their partner by asking and answering questions about the daily routine of some characters in some pictures (Appendix E). This information-gap activity was modeled for the students and they were also given a few key phrases in the target language to help them resolve any errors in communication that they might need to negotiate with only speaking in the target language. Each participant sat at a separate computer with a headset or they used their own electronic devices. The participants sat at least one person away from their partner. The participants then used the worksheet to go through the information-gap activity using only the audio feature.

The four oral tasks were administered throughout the duration of a month. The in-class presentational task was completed on the $9^{\text {th }}$ week of class and the online presentational oral task 
was completed on the $14^{\text {th }}$ week of class. The in-class interpersonal oral task was completed on the $10^{\text {th }}$ week of class and the online interpersonal oral task was completed on the $13^{\text {th }}$ week of class.

\section{Data Analysis}

The first six questions on the anxiety survey uses a Likert-scale in order to measure reported anxiety with 1 being the highest and 5 being the lowest except for in question 3 where the answers are reversed. Using the quantitative data collected from the surveys, we calculate the mean of all the answers for each oral task in each question. Using the mean, we analyze the data to see the relationship between task type, task medium (in-class vs. online) and anxiety. Responses from the open-ended questions were analyzed using thematic analysis (Braun \& Clarke, 2006). 


\section{CHAPTER IV: RESULTS}

The results of this study are completed and analyzed by computing the means and standard deviation from the surveys' responses for each oral task as well as thematically examining the responses from the open-ended questions in the survey. As suggested, the mix of qualitative and quantitative data demonstrates a more cohesive understanding of FLA manifested in the participants during the tasks they completed.

Each oral task had a varied number of participants that completed each task, as mentioned previously. Only six participants completed all four oral tasks; however, in order to get a general picture of the data, it was decided to treat the results from each task independently, which explains the disparity in number of participants from each task as shown in the results below. In order to facilitate the presentation and interpretation of the results, the researcher collapsed the five possible responses from the Liker-scale survey responses to three main categories: Strongly agree and agree, neutral, and strongly disagree and disagree.

The structure of this chapter is as follows. We will present quantitative and qualitative results for each research questions for this study. The chapter will end with a summary of main findings.

\section{Research question 1: How do participants' levels of FLA differ between presentational oral tasks completed in the classroom and presentational oral tasks completed in an online interface?}

In order to answer our first research question, we look at the results from the anxiety survey that students completed after the in-class presentational task and the online presentational task. Since each question in the survey asked something different, we offer a summary of results from each question. 
The first question on the survey looks at how participants perceive their anxiety during an oral task. Results from the presentational oral tasks appear in Figure 1. Overall, 56\% indicated that they feel nervous while completing an oral task as compared to $32 \%$ who reported not feeling nervous. In the in-class presentational oral task $(\mathrm{N}=13, \mathrm{M}=3.08, \mathrm{SD}=1.21), 47 \%$ of participants indicated that they feel nervous whereas $31 \%$ indicated they do not feel nervous. The in-class presentational oral task had the lowest number of students who felt nervous during the performance of the oral task out of all four task types.

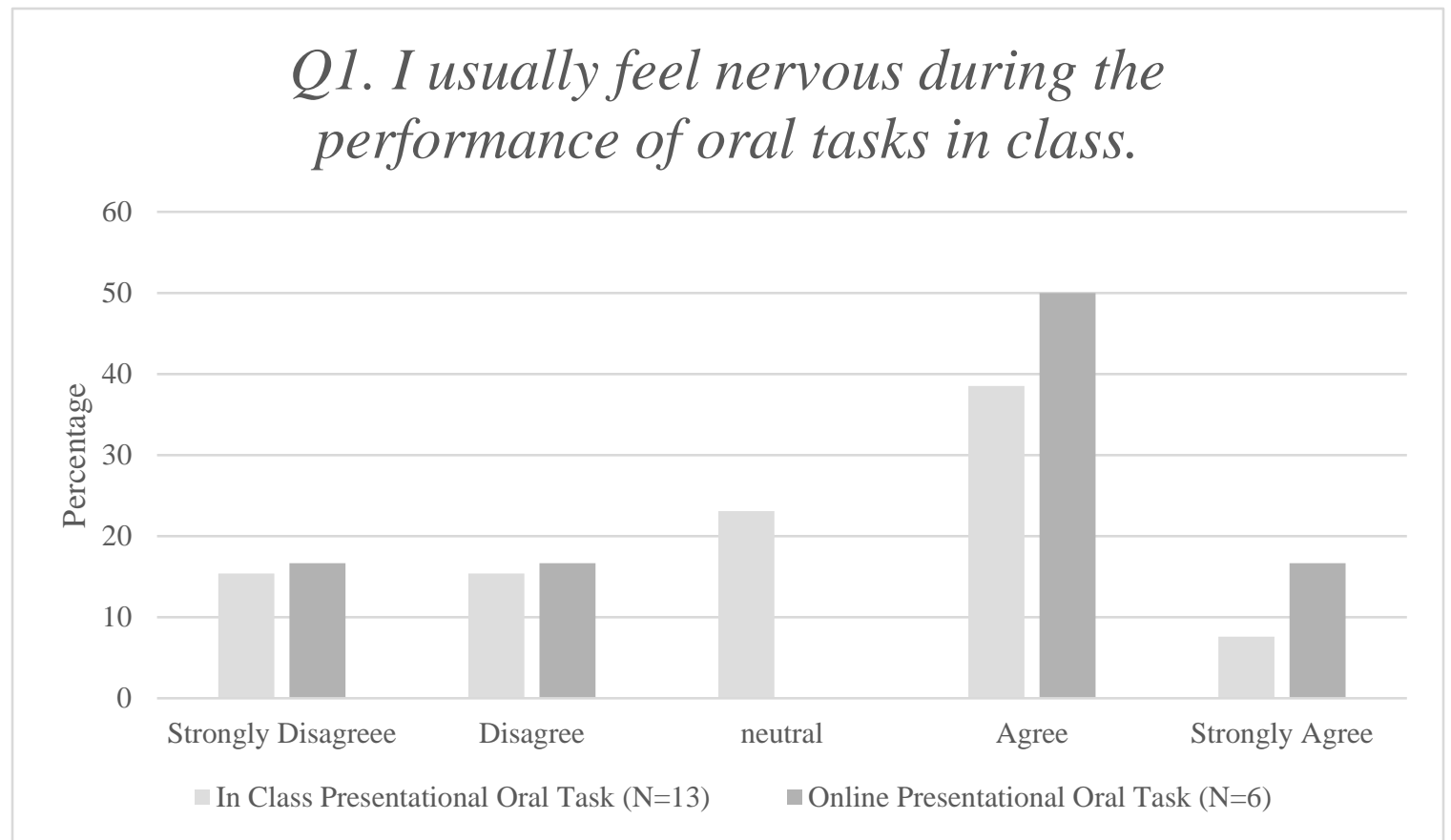

Figure 1. Responses to Q1, I usually feel nervous during the performance of oral tasks in class.

In the online presentational oral task $(\mathrm{N}=6, \mathrm{M}=3.33, \mathrm{SD}=1.37), 67 \%$ of participants indicated that they feel nervous while doing oral tasks whereas $33 \%$ indicated they do not feel nervous. The online presentational oral task had the highest percentage of participants across all four tasks who felt anxious while completing oral tasks. 
The second question asks if participants tremble when they need to speak in Spanish in front of their peers which relates the physical experience of anxiety to oral task performance and how anxiety affects the participants. Results from all presentational oral tasks for question 2 appear in Figure 2. Overall, $11.5 \%$ of participants agreed to trembling when they speak in Spanish either in front of classmates or with their online partner and $77 \%$ of participants indicated that they do not tremble while speaking in Spanish in front of other classmates or with their partner online. In the in-class presentational oral task $(\mathrm{N}=13, \mathrm{M}=2.33, \mathrm{SD}=.94), 23 \%$ of participants reported that they tremble while speaking in Spanish and 54\% of participants reported that they donot tremble while speaking in Spanish.

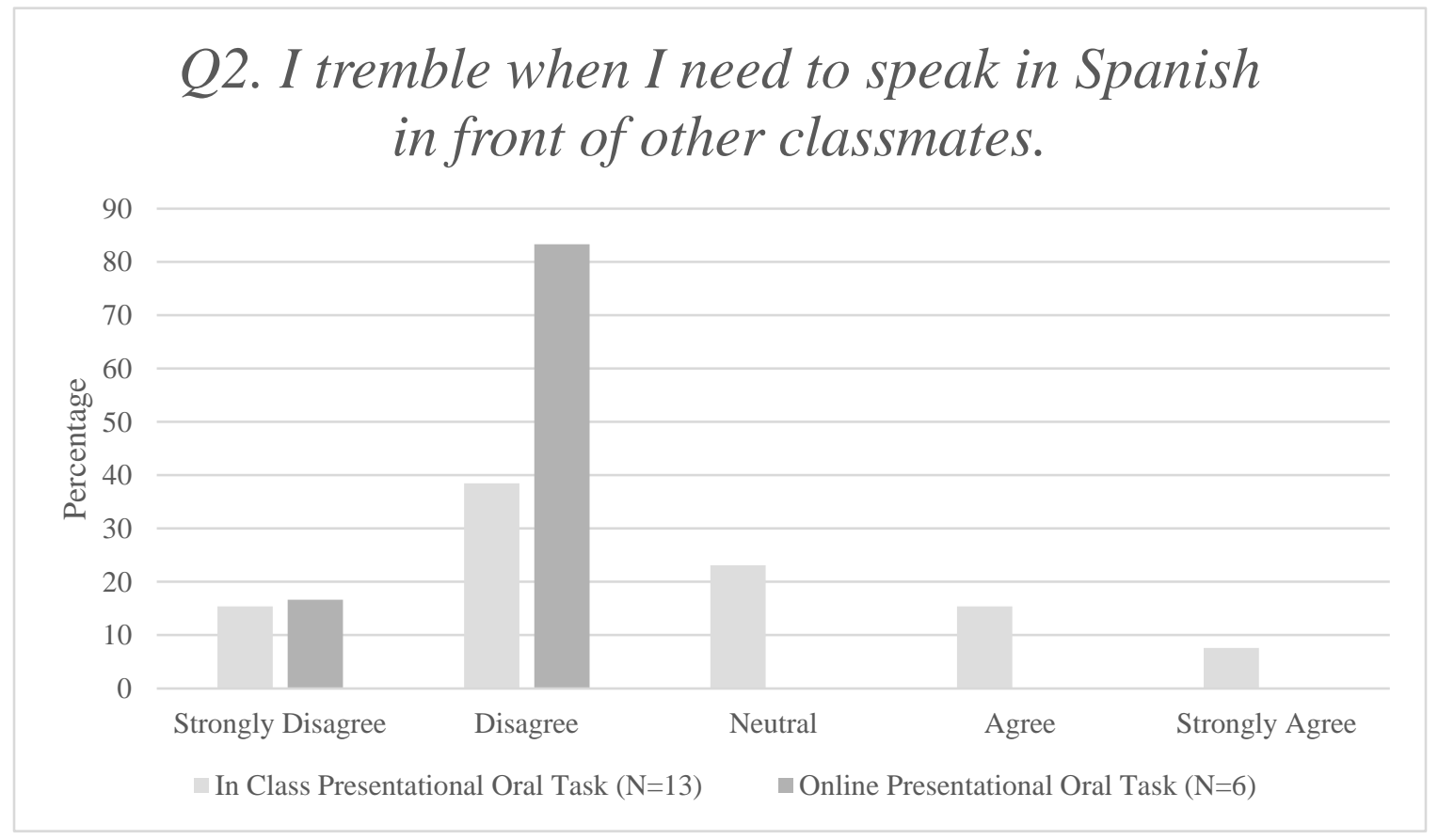

Figure 2. Responses to Q2, I tremble when I need to speak in Spanish in front of other classmates.

As for the online presentational oral task $(\mathrm{N}=6, \mathrm{M}=1.83, \mathrm{SD}=.37)$ none of participants reported that they tremble but all of them (100\%) reported that they do not tremble. The standard deviation (.37) is rather low meaning that there was little variance between answers. This data set 
represented the lowest reported effect of FLA in all the surveys. However, this is not surprising because the participants are not interacting with another speaker during the online presentational task.

Figure 3 reflects the data on how participants feel anxious because of the mistakes they make while doing oral tasks. This question relates to communication apprehension and fear of negative evaluation. Overall, $32 \%$ reported that they do not worried about making mistakes during the oral tasks while $60 \%$ worry about making a mistake. For the in-class presentational oral task $(\mathrm{N}=13, \mathrm{M}=2.62, \mathrm{SD}=1.15), 31 \%$ of the participants do not worried about making mistakes while $54 \%$ of participants said they worry about making mistakes.

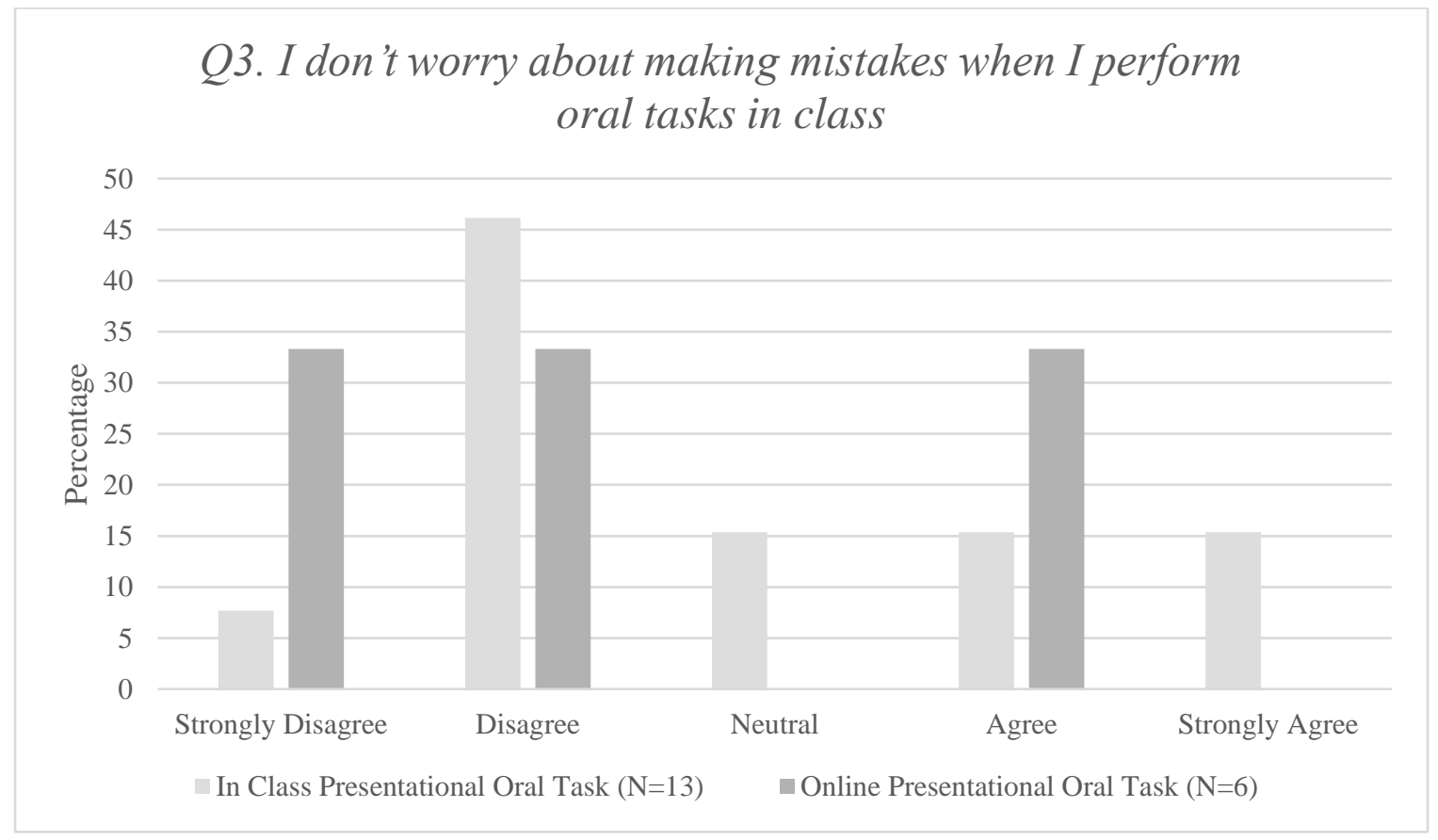

Figure 3. Responses to Q3, I don't worry about making mistakes when I perform oral tasks in class.

As for worrying about making mistakes during an online oral presentational task $(\mathrm{N}=6$, $\mathrm{M}=2.33, \mathrm{SD}=1.25)$, the majority (67\%) claimed they worry about making mistakes while $33 \%$ do not worry. Overall, participants seem to worry about making mistakes during each of these two 
presentational tasks. The in-class task, however, received comments that related to the task "not being graded so [participants] don't mind messing up", whereas the online task made participants feel that they "do not have enough time to think through what [they] want to say." The level of anxiety about making mistakes increases when there is more pressure for a grade and there is a visible time limit.

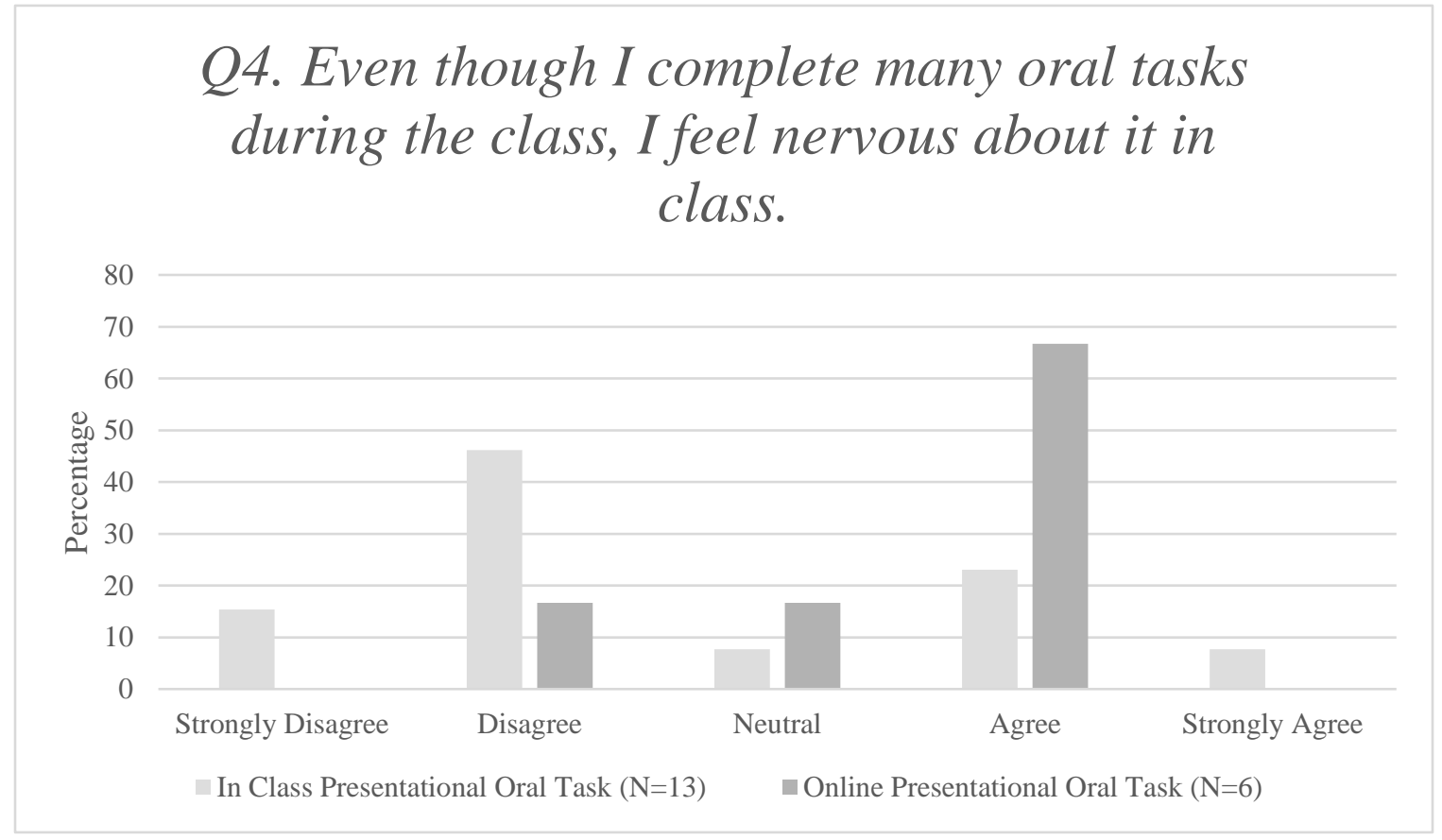

Figure 4. Responses to Q4, Even though I complete many oral tasks during the class, I feel nervous about it in class.

In Figure 4, the data reports results from a question that asked participants about feeling nervous despite the familiarity with oral tasks. This question is removing the novelty of the task to observe whether participants feel nervous for doing something they do regularly. Overall, $48 \%$ of participants indicated they are nervous even though they have completed many tasks in class, and $39 \%$ reported that they do not feel nervous. In the in-class presentational oral task $(\mathrm{N}=13, \mathrm{M}=2.62$, $\mathrm{SD}=.58), 30 \%$ indicated that they feel nervous while $62 \%$ reported that they disagreed or strongly 
disagreed with the statement. The in-class presentational oral task had the highest percentage of participants reporting that they do not feel nervous.

Due to the similarities between question 1 and 4, it is important to compare the results between the two questions. The difference between question 1 and question 4 for the in-class presentational task is startling as only $46 \%$ of participants reported feeling nervous when performing oral tasks in question 1 as compared to the $30 \%$ in question 4 . In question 1 , there was also $22 \%$ of participants saying they feel neutral about their level on anxiety during oral tasks as compared to $8 \%$ in question 4 . The results are more similar to a standard bell curve for question 1 than in question 4 . These differences seem to show that when students were reminded that the task is similar to those they have already done in class, their level of anxiety diminished.

The online presentational oral task $(\mathrm{N}=6, \mathrm{M}=3.5, \mathrm{SD}=.76)$ had $17 \%$ indicated not feeling anxious as opposed to $67 \%$ who mentioned they were nervous. Although participants reported feeling nervous, there were no participants that selected 'strongly disagree' to the statement, 'Even though we complete many oral tasks during the class, I feel anxious during oral tasks'. This interesting fact indicates that although the great majority of students still claim anxiety during oral tasks, when reminded that these oral tasks are similar to many of the oral activities they do in class, their perception of their anxiety level is not as negative as when asked to think about oral tasks and FLA in general.

Contrary to the results from the in-class presentational oral tasks, in the online presentational task when we compare levels of anxiety from participants' responses in question 1 and question 4 , we see the same level of anxiety since $67 \%$ of participants in both tasks claimed to be nervous regardless of the familiarity of the task. Another interesting finding in making this comparison between question 1 and 4 was that in question 1, participants either agreed or disagreed 
with the statement but none were neutral with regards to levels on anxiety. In question 4, 17\% of students said they were not nervous but $16 \%$ were somehow neutral. The online interface seems to trigger stronger opinions in students' perceptions of anxiety to the extent that the fact that students are aware that they do many oral tasks in the classroom (question 4), this fact does not seem to have as much of an impact in evaluating one's anxiety as it does the fact the activity is done online.

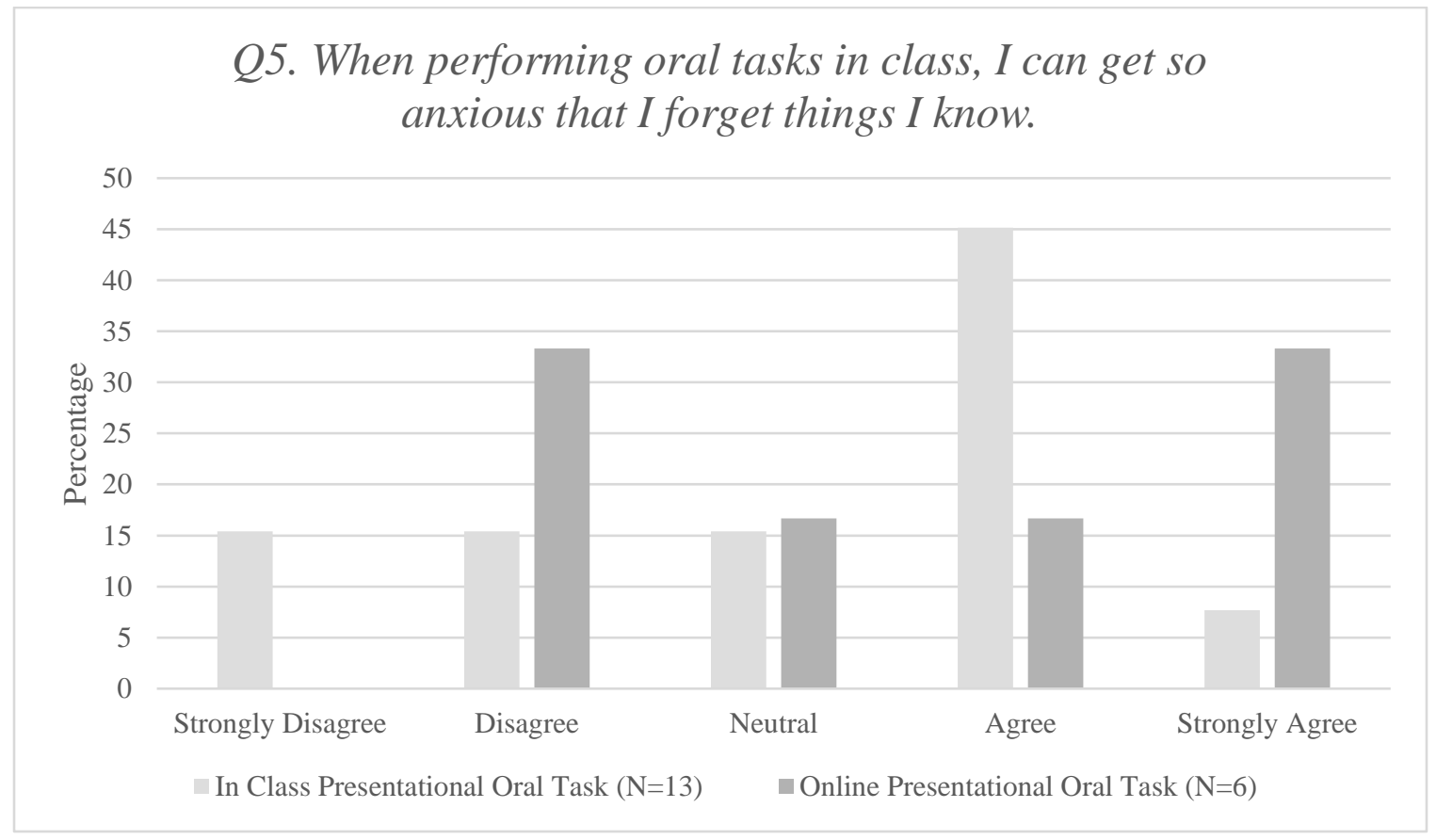

Figure 5. Responses to Q5, When performing oral tasks in class, I can get so anxious that I forget things I know.

The next question in the anxiety questionnaire asked whether anxiety levels impact forgetfulness when completing oral tasks. This question looks at how FLA can block cognitive processing and vocabulary retrieval. The answers to this statement can be interpreted in different ways, as it could mean that students were not nervous enough to forget words, that they were nervous, but they do not forget words during a task, or that they were not nervous. For this reason, 
this data must be compared to those of question 1 as to understand if students feel nervous during oral tasks and how FLA makes them react.

Figure 5 reflects the percentage of participants who "when performing oral tasks in class/online, I can get so anxious that I forget things I know" during the two different oral tasks. Overall, $51 \%$ reported feeling so anxious that they forget things they knew while $32 \%$ reported they disagreed with this statement. Regarding the in-class presentational oral task $(\mathrm{N}=13, \mathrm{M}=3.15$, $\mathrm{SD}=1.23)$, the majority of participants $(53 \%)$ feel so nervous that they forget information while $31 \%$ reported not feeling so nervous.

As for the online presentational oral task $(\mathrm{N}=6, \mathrm{M}=3.5, \mathrm{SD}=1.26), 33 \%$ reported not feeling so anxious that they forget things they know while $50 \%$ felt that they either agreed or strongly agreed with feeling so nervous that they forget information while completing an oral task. In both the in-class $(53 \%)$ and the online $(50 \%)$ presentational tasks over half of the participants indicated they forget information due to FLA. These results show that the medium in which the oral task takes places does not seem to have an effect here.

Regardless of whether the task is done online or in class, during presentational tasks students claim their level of anxiety negatively impacts their proficiency since they tend to forget things. Participants claimed that they "do not like public speaking" in the in-class task while in the online task a participant openly commented that they do "get so nervous they forget Spanish." One difference from the online task is that anxiety is lower when "answering simple questions" and higher when participants "have to expand" on their answers. This could be that since the online task had short prompts that anxiety was lower when it came to forgetting things.

In comparing results from question 1 and question 5, we see that when assessing anxiety levels in general the medium makes a difference since the online interface triggered higher levels 
of anxiety (67\%) as compared to in-class presentational tasks (47\%). It is when students are asked to reflect on how anxiety impacts performance such as forgetting what to say (question 4) that we see that overall half of participants agree that their anxiety is responsible for their forgetfulness during presentational oral tasks regardless of the medium in which these oral tasks are completed.

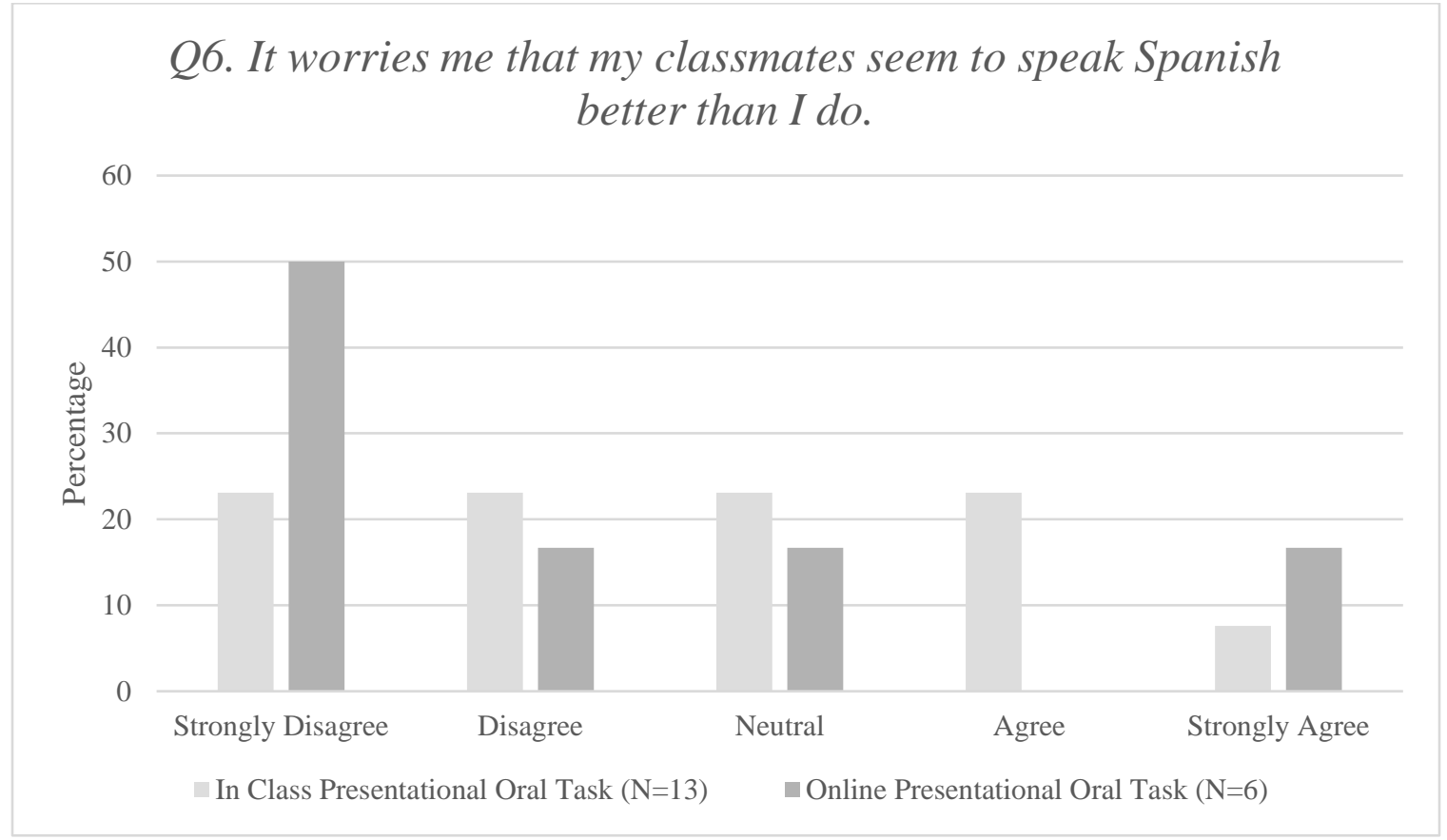

Figure 6. Responses to Q6, It worries me that my classmates seem to speak Spanish better than I do.

The final question in the survey looks at how FLA is manifested within students when they are comparing themselves to their peers. This question looks at FLA through fear of negative evaluation by their peers as they may believe that they are being judged by their level of Spanish as compared to other students in their class. The results for this question for the two presentational tasks are presented in figure 6 . Overall, $24 \%$ indicated that it worries them that their partner may speaks better Spanish than them, while 56\% reported that it does not worry them. Regarding the in-class presentational class $(\mathrm{N}=13, \mathrm{M}=2.69, \mathrm{SD}=1.26), 46 \%$ responded that they are not worried about their peers speaking better than them, while $31 \%$ reported that they worry. 
The results from the online oral presentational task $(\mathrm{N}=6, \mathrm{M}=2.17, \mathrm{SD}=1.46)$ showed that $17 \%$ of the participants reported that it worries them that their classmates speak better Spanish than they do, while $6 \%$ indicated that it does not worry them. Although overall levels of anxiety related to fear of evaluation from their peers were not very high as indicated by these results (17\%: online, 31\%: in-class), we see that the presentational task in the classroom worried students slightly more.

During the in-class presentational task, students were placed in groups and each student had to present their family to their classmates as compared to the online presentational task which had no audience since the student completed the oral task alone in front of the computer. Therefore, fear of evaluation by their peers as a variable is removed in the online task although students may still fear negative evaluation by the instructor since this task was being graded by the instructor. Even though there was a difference between the two mediums, participants overall reported feeling comfortable speaking with their classmates. Participants in the in-class task reported feeling "not super nervous because it was with a few students who were in the same boat as me" and "not as nervous because it was only with two other students and not the whole class." However, the online task was greeted with more comfort because there was no one listening to the speaker. Participants feel "more comfortable with the oral tasks online" because "it's only me and a computer."

In conclusion, a large majority of the data were very similar in percentages. It seems that anxiety is lower for online tasks when it relates to communication apprehension and fear of negative evaluation. The higher anxiety for the in-class task may be explained by the conditions of the task itself. The participants completed a task in front of other students while there was no audience for the online task. The other finding shows that there is an increased level of anxiety when looking at data relating to test anxiety and general anxiety with online tasks. Since the online 
task was graded, there may be more anxiety related to it, which is shown in the participants' comments.

\section{Research question 2: How do participants' levels of FLA differ between interpersonal oral tasks completed in the classroom and interpersonal oral tasks completed in an online interface?}

Overall, $52 \%$ of participants reported feeling anxious during interpersonal oral tasks while $34 \%$ did not in regard to the question on feeling anxious in oral tasks. The results for both the inclass and online interpersonal oral tasks are found in Figure 7. In the in-class interpersonal oral task $(\mathrm{N}=22, \mathrm{M}=3.27, \mathrm{SD}=1.35), 50 \%$ of the participants indicated that they feel anxious during oral tasks while $32 \%$ of the participants said they do not feel anxious.

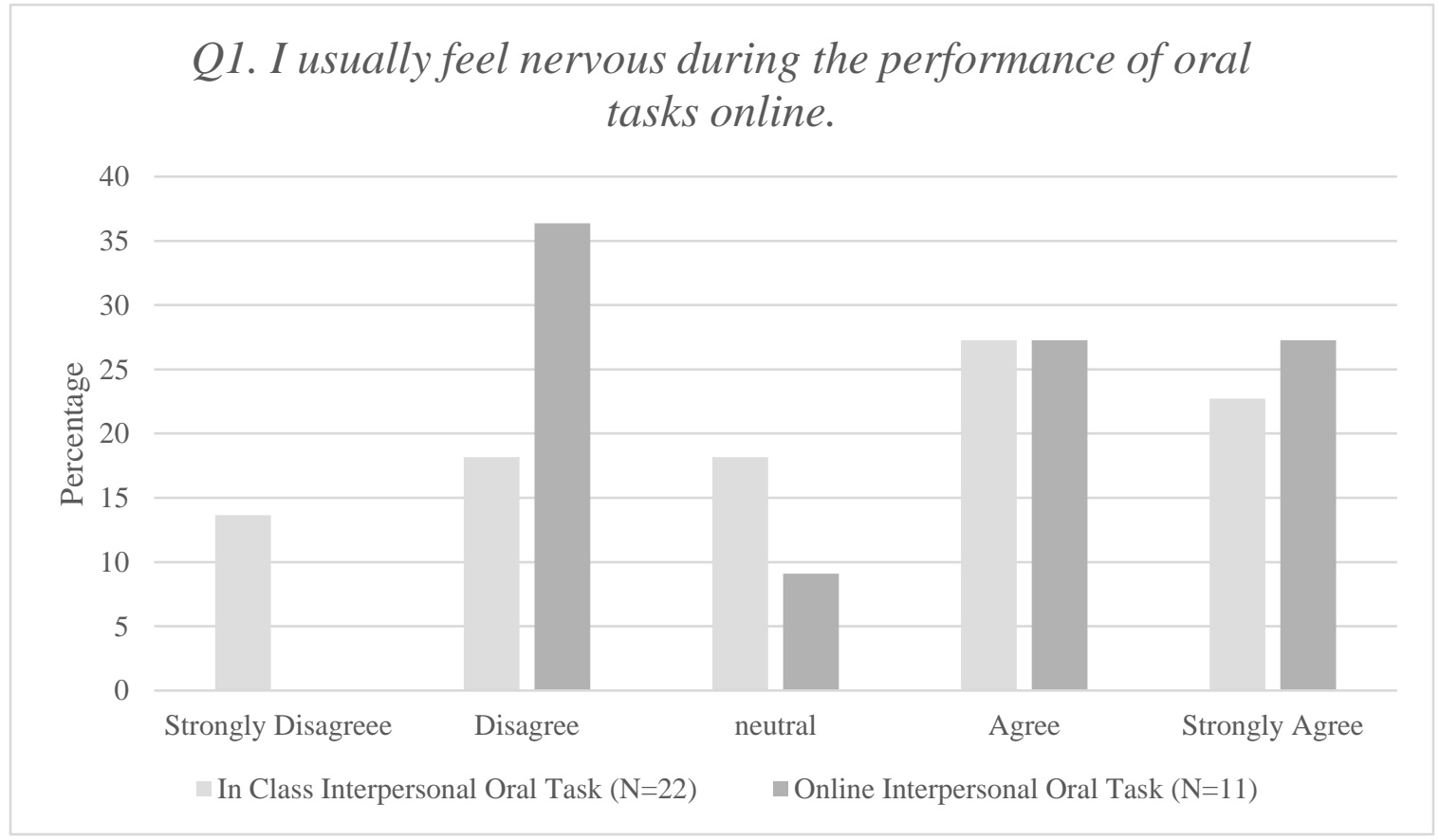

Figure 7. Responses to Q1, I usually feel nervous during the performance of oral tasks online.

As for the online interpersonal oral task $(\mathrm{N}=11, \mathrm{M}=3.45, \mathrm{SD}=1.23), 54 \%$ of participants indicated that they feel anxious and $36 \%$ of participants indicated that they do not feel anxious 
during oral tasks. These results suggest that in-class tasks had slightly lower anxiety when it comes to interpersonal tasks. Visually, in-class tasks support a slightly normal bell-curve that is not apparent in online tasks. There is a lack of participants strongly disagreeing with the prompt for online tasks however $27 \%$ of participants strongly agreed with feeling anxiety.

With regards to the question about trembling when speaking in front of classmates, 27\% of participants agreed and $45 \%$ of participants disagreed. The results for both the in-class and online interpersonal oral tasks are found in Figure 8.

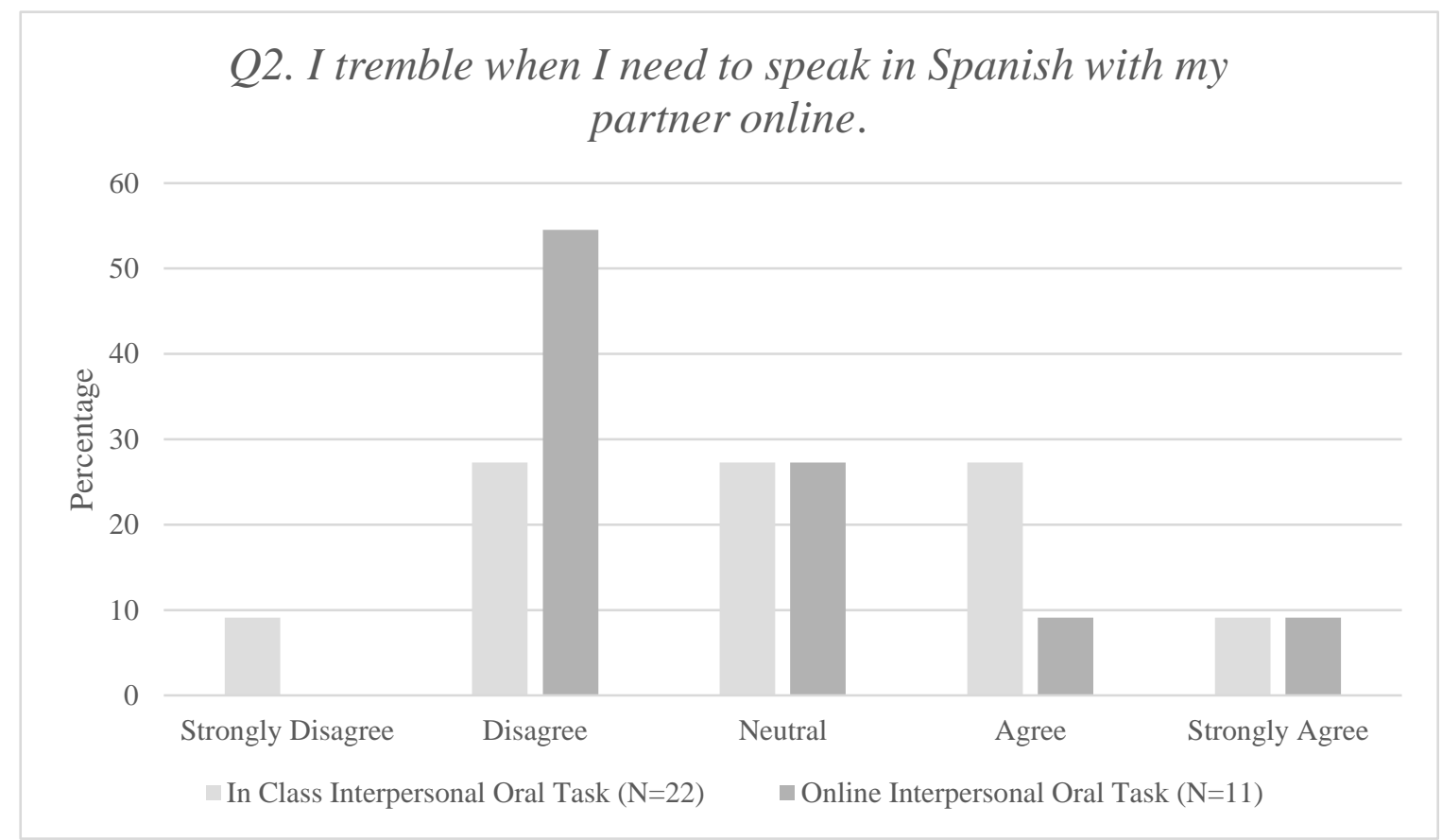

Figure 8. Responses to Q2, I tremble when I need to speak in Spanish with my partner online.

The in-class interpersonal oral task $(\mathrm{N}=11, \mathrm{M}=3, \mathrm{SD}=1.13)$ is quite evenly distributed among all the participants. The data shows that $36 \%$ of the participants indicate that they tremble while speaking in Spanish in front of their partner while 36\% do not tremble. This data reflects neither a positive or negative relationship to the question and it is the only time that this happens in any data set. 
As for the online interpersonal oral task $(\mathrm{N}=11, \mathrm{M}=2.73 \mathrm{SD}=.96)$, the majority of the participants $(55 \%)$ reported that they do not tremble in front of their classmates while $19 \%$ indicated that they tremble. The higher levels of anxiety from the interpersonal task may come from being able to visually see their partner. During the online task, the partners, although in the same room, were only using the audio feature, therefore they were not seeing each other.

Fear of making mistakes triggered some interesting results: $14 \%$ indicated they do not worry about making mistakes while $68 \%$ worry about making mistakes during oral tasks. The results for both the in-class and online interpersonal oral tasks are found in Figure 9.

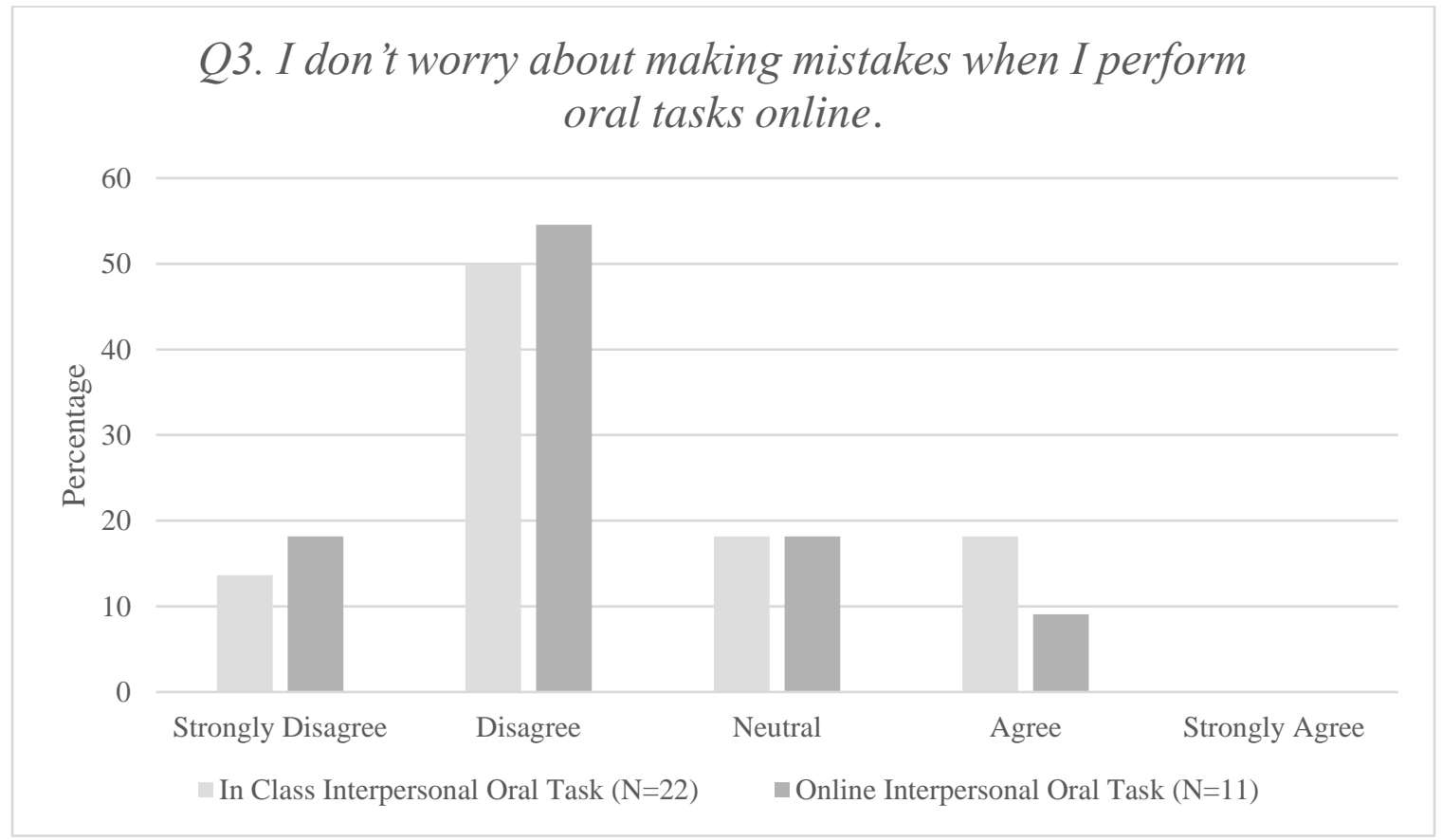

Figure 9. Responses to Q3, I don't worry about making mistakes when I perform oral tasks online.

The in-class interpersonal oral task $(\mathrm{N}=22, \mathrm{M}=2.41, \mathrm{SD}=.94)$ had $64 \%$ of participants who worry about making mistakes while speaking with a partner and $18 \%$ that indicated they do not worry about mistakes. One participant mentioned that the lower anxiety in the in-class 
interpersonal task may be attributed to the fact that there is "never really a consequence in class if [they] say something wrong.”

During the online interpersonal task $(\mathrm{N}=11, \mathrm{M}=2.18, \mathrm{SD}=2.18)$, an overwhelming majority (73\%) of participants reported a feeling of worry or anxiousness about making mistakes. Only $9 \%$ agreed to not feeling worried about making mistakes during an oral task. Overall, participants reported high levels of anxiety in both in-class and online about making mistakes, however the in-class task had slightly less anxiety associated with it. One difference with this task is that students had time to write down questions before asking them in the in-class task. One participant mentioned "if I have time to write down the words [the task] is easier." In the online task "testing anxiety" was mentioned explicitly as a reason to why they were nervous.

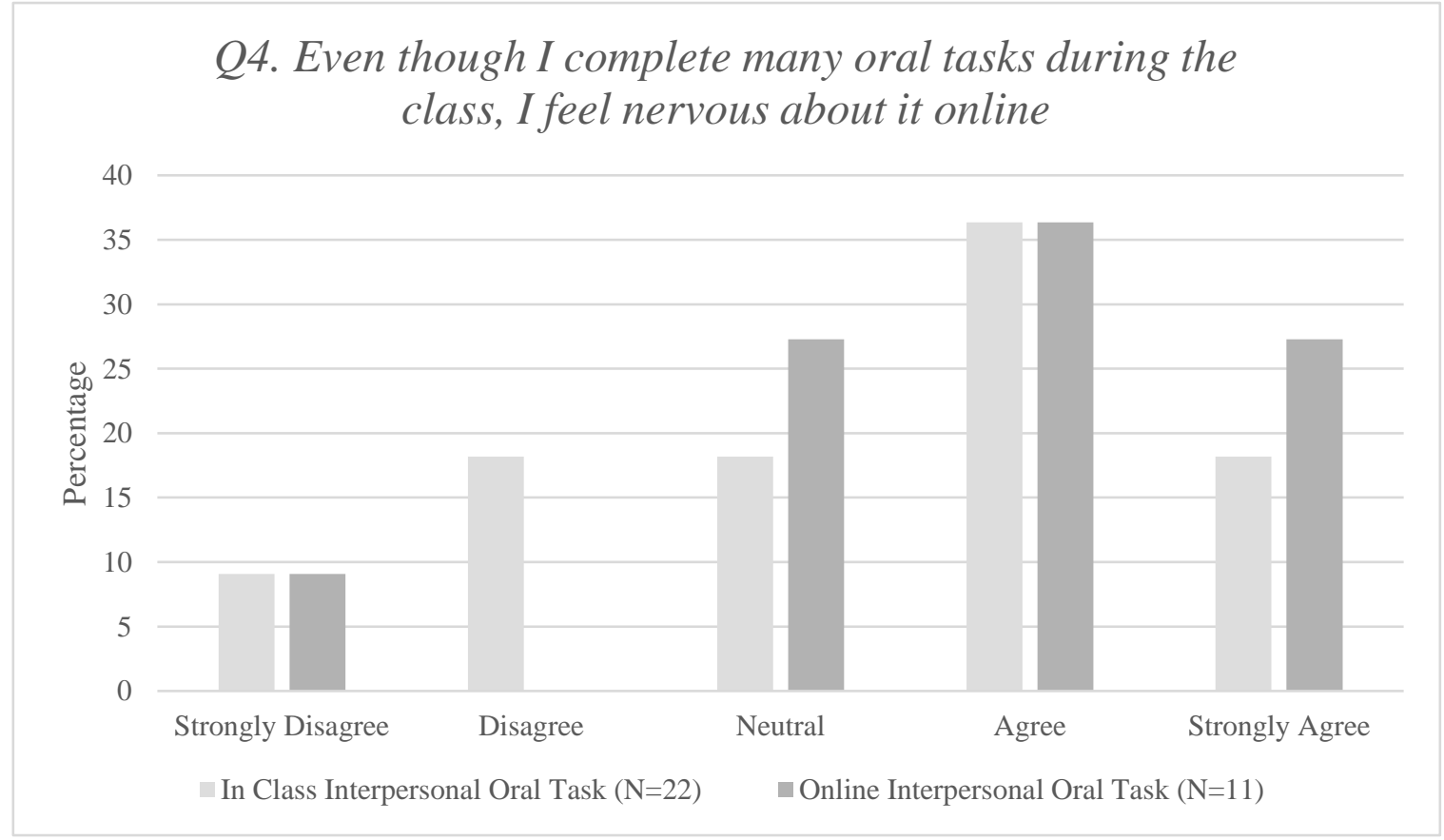

Figure 10. Responses to Q4, Even though I complete many oral tasks during the class, I feel nervous about it online. 
The question about the familiarity of the task and levels of anxiety resulted in $59 \%$ of participants indicating they feel nervous and $18 \%$ who do not. The results for both the in-class and online interpersonal oral tasks are found in Figure 10.

For the in-class interpersonal oral task $(\mathrm{N}=22, \mathrm{M}=3.36, \mathrm{SD}=1.23)$, the majority of participants (55\%) said they are nervous even though they are familiar with oral tasks, and only $27 \%$ indicated they do not feel nervous.

In regard to the online interpersonal oral task $(\mathrm{N}=11, \mathrm{M}=3.73, \mathrm{SD}=1.14), 9 \%$ indicated not feeling nervous during online oral tasks while the majority of participants (67\%) reported feeling nervous. Once again, overall, both tasks had high anxiety levels associated with feeling nervous during oral tasks although they have completed many oral activities in the classroom. However, there was a lower percentage of participants who felt this way in the in-class tasks. No comments from participants were made about the repetition or completing more exercises in the classroom to feel less anxious.

These results for both the in-class and the online interpersonal tasks are more or less reflective of the results from question 1 where $50 \%$ (in class) and 55\% (online) of the participants reported feeling anxious. In this question about the familiarity of the task, we see similar results with the majority of students feeling quite nervous (55\% in class, $63 \%$ online) as well. The fact that participants were asked to reflect on the familiarity of the interpersonal task did not seem to impact the level of anxiety as students feel anxious about oral tasks in general, although slightly more with online interpersonal oral tasks.

These results, however, slightly different from the results from the presentational tasks where for this question, the medium in which the task was completed seemed to have made a difference. There, the in-class presentational task triggered less anxiety (30\%) than the online 
presentational task (67\%). This increase in the online presentational tasks overall demonstrates that even though participants practice speaking with a partner, their anxiety does not decrease as they do when they practice presenting information

Results from the question that relates FLA and language performance as in the case of forgetting information when one is anxious show that $75 \%$ of participants reported they get anxious and forget information while $9 \%$ do not. The results for both the in-class and online interpersonal oral tasks are found in Figure 11. The in-class interpersonal oral task ( $N=22, M=3.95$, $\mathrm{SD}=1.02$ ) showed that $9 \%$ of participants responded not feeling so anxious they forget information while an overwhelming majority of participants (77\%) agreed or strongly agreed with the statement.

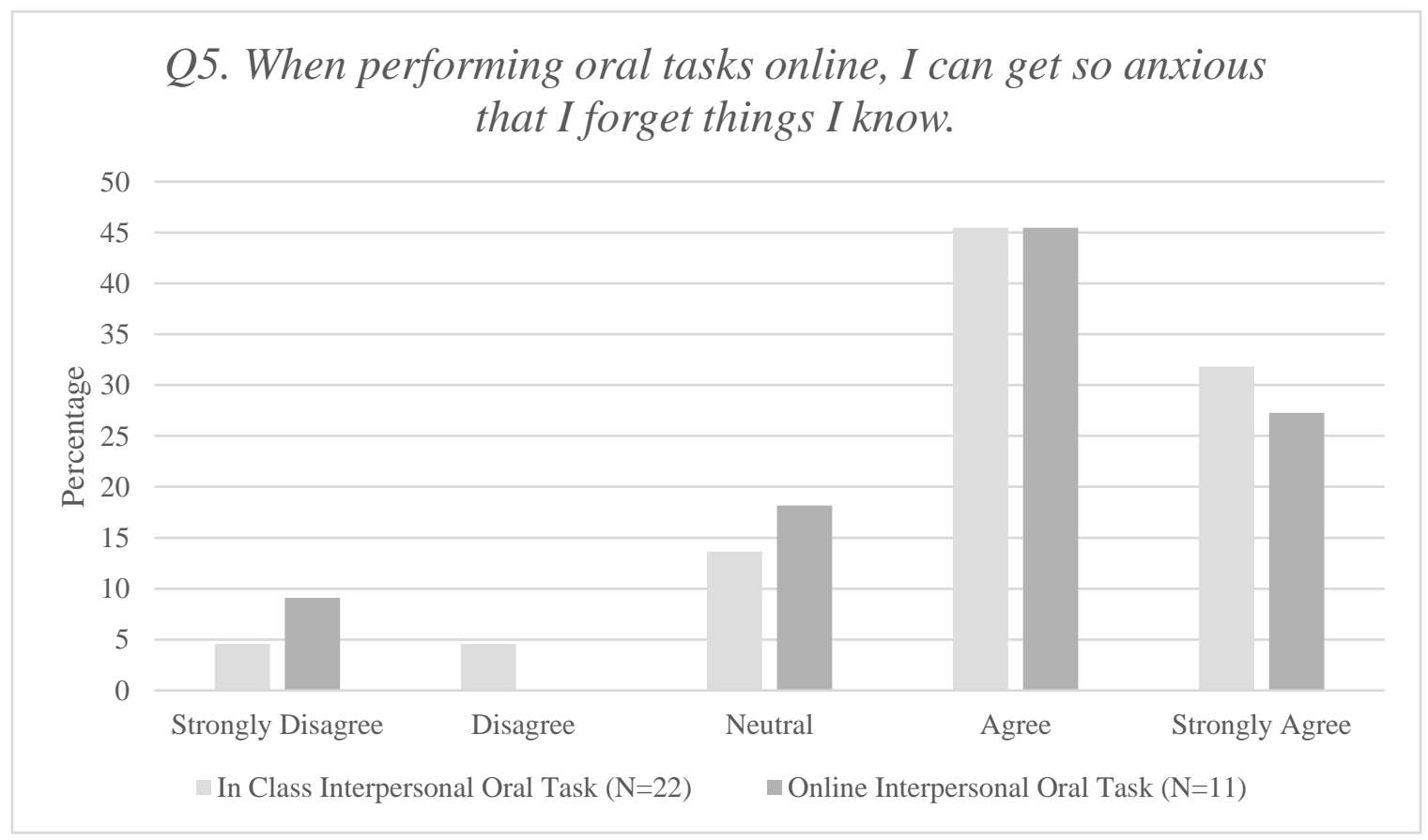

Figure 11. Responses to Q5, When performing oral tasks online, I can get so anxious that I forget things I know.

Regarding online interpersonal oral task $(\mathrm{N}=11, \mathrm{M}=2.18, \mathrm{SD}=.83)$ and forgetting information due to FLA, 9\% disagreed that their FLA influences their performance while 73\% 
believe their anxiety place a role in forgetting what they want to say. Overall, regardless of the medium with interpersonal tasks, the great majority of participants claim that their level of anxiety is an obstacle to their language performance because they forget things as they complete oral tasks. One comment made by a participant in the online task mentioned that "test anxiety makes [them] forget things." However, a similar level of anxiety was triggered by the graded online interpersonal task $(73 \%)$ as well as by the in-class non-graded task (77\%). It is interesting to note, however, that when participants were asked to reflect on how their anxiety impacts their performance (75\%), their level of perceived anxiety is much higher than when they were asked whether completing oral tasks made them feel anxious in question $1(52 \%)$. .

Finally, the question that focuses on fear of evaluation from their peers showed that $41 \%$ of participants report feeling nervous while $43 \%$ do not. The results for both the in-class and online interpersonal oral tasks are found in Figure 12.

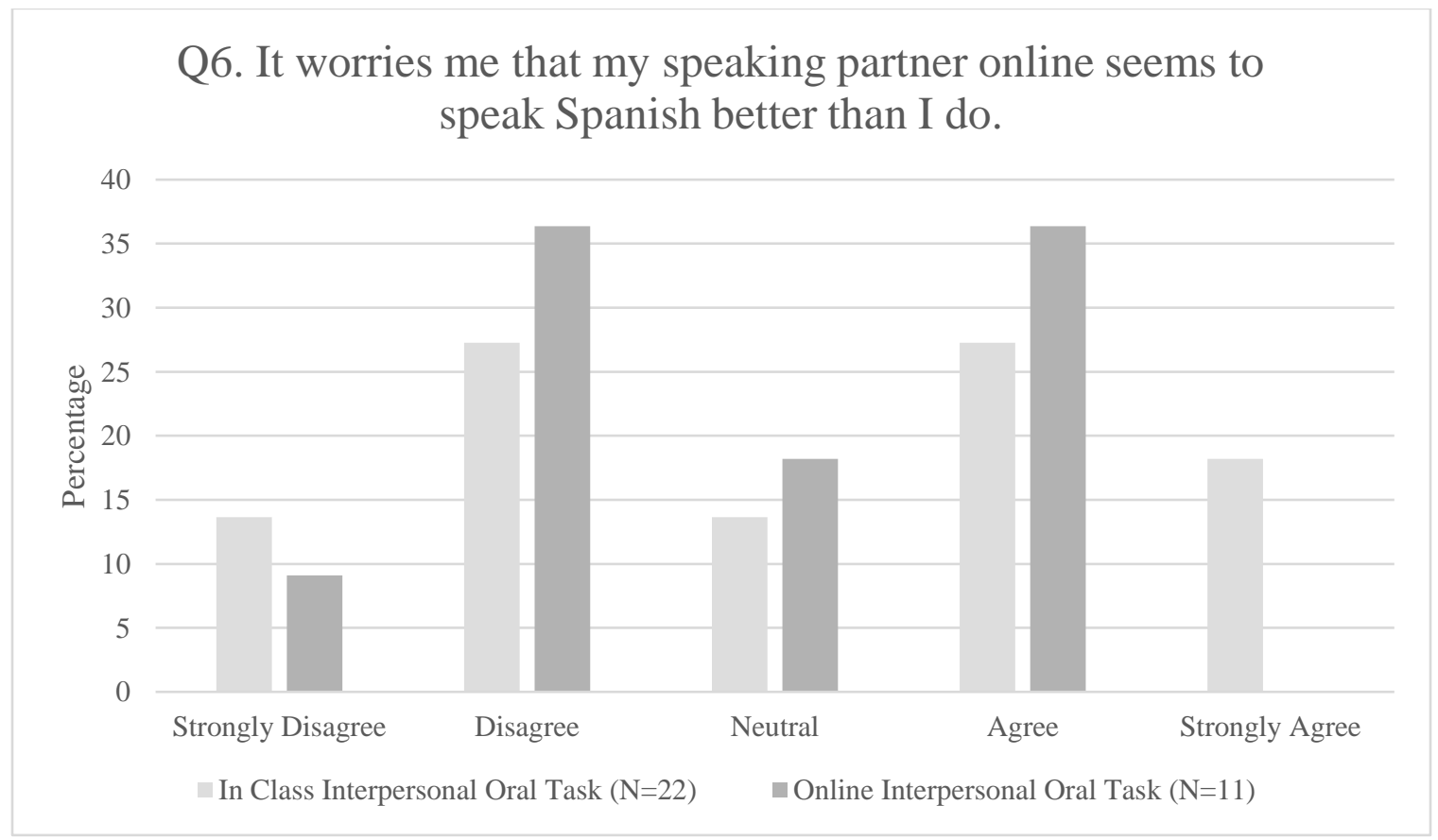

Figure 12. Responses to Q6, It worries me that my speaking partner online seems to speak Spanish better than I do. 
As for results for the in-class interpersonal oral task $(\mathrm{N}=22, \mathrm{M}=3.09, \mathrm{SD}=1.35), 41 \%$ reported not being worried and $46 \%$ indicated that worry about their classmates speaking better. Fear of negative evaluation by a partner was brought explicitly for in-class interperosnal tasks with participants saying they "felt nervous and uncomfortable because [their] partner knows more Spanish," "think other students are better" and that making errors made them "feel more nervous to speak to my partner."

The responses for the online interpersonal oral task $(\mathrm{N}=11, \mathrm{M}=2.82, \mathrm{SD}=1.03)$ showed that a large portion of participants $46 \%$ strongly disagreed or disagreed with feeling nervous or worried that their partner speaks better than they do while 36\% agreed with feeling worried because of their partner's oral abilities. Overall, the results indicate that participants are equally divided between those who fear peer evaluation and those who do not, regardless of the medium in which the interpersonal task is completed.

In conclusion, interpersonal oral tasks completed in the classroom have, overall, lower levels of anxiety related to them, although participants seem to display more physical signs of anxiety such as trembling and are more worry about making mistakes in the in-class interpersonal tasks than in the online tasks. During these interpersonal tasks, regardless of the medium, students mention their FLA impacts their language performance in a way that they forget what they want to say. Finally, fear of evaluation is assessed in a very balanced way with almost the same number of participants worrying about being compared with others and the other half not worrying.

It is also worth noting that presentational and interpersonal tasks impact anxiety in some different ways. First, participants in this study, regardless of whether the task was interpersonal or presentational or whether it was in-class or online claim they worry about making mistakes. Similarly, both types of tasks also resulted in higher levels of anxiety associated with forgetfulness 
although, interpersonal tasks trigger higher levels of anxiety. In addition, students are aware that completing oral tasks impacts their anxiety level, regardless of task type. The main difference between presentational and interpersonal tasks resides in the fact that during interpersonal tasks students are more worried about making mistakes than during presentational tasks. Similarly, when examining physical consequences of anxiety such as trembling, interpersonal tasks seem to cause more anxiety than presentational tasks.

\section{Research question 3: What factors are associated with different levels of FLA in oral tasks completed in the classroom and oral tasks completed in an online interface?}

In order to answer this question, we take two approaches. On the one hand, we have calculated the average means from the students' surveys data for the online and in-class tasks as seen in Table 1 below. On the other hand, we have looked at the responses from the open-ended responses from the survey and identified possible factors or themes that contribute to FLA in online and in-class oral tasks.

\section{Table 1}

Means of Survey Questions between Online and In-Class Tasks

\begin{tabular}{lllllllllllll}
\hline & $Q 1$ & & $Q 2$ & & $Q 3$ & & $Q 4$ & & $Q 5$ & & $Q 6$ \\
\hline \multirow{2}{*}{ Online } & $\mathrm{A}$ & $\mathrm{D}$ & $\mathrm{A}$ & $\mathrm{D}$ & $\mathrm{A}$ & $\mathrm{D}$ & $\mathrm{A}$ & $\mathrm{D}$ & $\mathrm{A}$ & $\mathrm{D}$ & $\mathrm{A}$ & $\mathrm{D}$ \\
& 61 & 35 & 9 & 77 & 21 & 70 & 65 & 13 & 61 & 21 & 27 & 56 \\
In-class & 48 & 31 & 30 & 45 & 25 & 59 & 42 & 44 & 65 & 20 & 38 & 44 \\
\hline
\end{tabular}

The numeric data from Table 1 shows the means of the students' answers for agreement (A) and disagreement (D) for each of the survey questions (Q). The table shows that overall anxiety during oral tasks, questions 1 and 4, is higher during online tasks (Q1: 61\%, Q4: 65\%). Anxiety associated with making mistakes during tasks is also high for online tasks (Q3: 70\%). As for forgetting information due to nervousness, there is no large difference between the medium used 
(Q5: Online 61\%, In-Class 65\%). Online tasks have less anxiety associated with them for physical responses (trembling) and worrying that their partner speaks better Spanish than they do (Q2: 9\%, Q6: 27\%).

Responses from the open questions in the survey also allows us to draw some conclusions about how the medium in which the oral task is completed impacts FLA levels. In examining students' responses, four themes were found that either increased or decreased levels of anxiety during oral tasks, (1) peer comparison, (2) test anxiety, (3) fear of negative evaluation and (4) preparedness.

\section{Peer Comparison}

Observing fear of negative evaluation and peer comparison between in-class and online tasks (Q6), online tasks had lower anxiety (27\%) as compared to in-class tasks (38\%). Interestingly enough, participants did not report a huge difference between online and presentational tasks, but rather there was a clear difference in task type (presentational vs. interpersonal). This is the only question from the survey that looks directly at peer interaction.

The in class presentational task received comments such as "not [feeling] super nervous because it was with a few students who were in the same boat as me" and "[feeling] more comfortable because it was my peers." However, the in-class interpersonal task received many negative comments such as "I am afraid I will mess up and people will judge me" and having everyone look at you while you are trying to remember what to say is intimidating." In line with question 6, one comment read "I felt nervous and uncomfortable because my partner knows more Spanish than me." This fear of negative evaluation is more apparent in the in-class task.

The opposite is present for the online tasks. For the online presentational tasks, participants felt less nervous during the presentational task. Some examples of comments are "It is more 
comforting to be able to do the task without the person sitting right next to you" and "I feel more comfortable with the oral tasks online. [Online tasks] make me feel less pressure as it is just me and a computer." The data on peer comparison and "It worries me that my classmates/speaking partner online seems to speak better Spanish than I do" also is in agreement that completing these tasks alone can help reduce anxiety as the online presentational oral task had a lower score in anxiety than the in-class presentational oral task.

In summary, it seems that online tasks have less anxiety associated with negative evaluation from peers. Given the comments made by the participants, one factor for this is that during online tasks students feel that they are more removed from their classmates so there is not peer-judgement.

\section{Testing Anxiety}

Horwitz et al. (1986) defined test anxiety as related to the fear of failure. Overall, participants reported lower levels of anxiety for the in-class oral tasks (59\%) than the online oral tasks $(70 \%)$ when looking at making mistakes. Unlike peer comparison, there was no difference between task type, asserting that the anxiety came from the medium used.

This group of comments specifically looked at when participants mentioned a fear of failure, a grade or the time limit involved in the task. Grading and time limit were put into testing anxiety as they are typical factors that come into play when a student has to take a test. One common factor among in-class and online tasks is that a time limit can increase anxiety. An inclass commenter mentioned he "felt rushed" because of the time limit placed in the activity by the instructor, while an online task commenter said, "I do not have the time to think through what I want to say." The online interpersonal task had a maximum 8-minutes recording time and still participants felt they needed more time to finish. As for asking questions during interpersonal 
tasks, one comment read "when I have time to write it down and see the words it is easier," which relates to the flexibility of in-class tasks. The tasks completed in class allotted extra time if need be, so students take advantage of this time to help prepare their output.

However, there was a disparity between in-class and online tasks for making mistakes. For example, in-class presentational oral task from one participant read that the task was "not being graded so I did not mind messing up," and for the in-class interpersonal task one declared that "there is never really a consequence if we say something wrong." In contrast, the online tasks had participants feeling "more nervous because this is for a grade." Looking at this, and the question that directly addressed mistakes, (Q3) “I don’t worry about making mistakes while I perform oral tasks in class/online," participants felt more anxious about making mistakes online than they did in the classroom.

\section{Communication Apprehension}

Communication apprehension is generally defined as fear of speaking with others. Manifestations of that fear may come in different ways such as nervousness, and forgetting information, and freezing up. Anxiety can also manifest through physical symptoms such as trembling. Overall, trembling was not a weighty feature in neither in-class (30\%) nor online (9\%), however, online tasks had prominently lower score associated with physical symptoms.

The in-class presentational class only had one comment that said the participant was “nervous because I am shy, and I don't like public speaking." As for the in-class interpersonal task, one participant said that they "forget a lot of words" another said that they are "more nervous while answering questions because I forget things that I know," which is very similar to a third response of "while answering questions I have forgotten everything." This aligns with the high levels of anxiety in in-class tasks found in Q5 (65\%). One participant mentions that they got 
"nervous when trying to ask the right question." Finally, for in-class interpersonal tasks, a participant said that "sometimes I just freeze on tests and oral exercises".

For the online presentational oral task, one participant said they "get nervous and forget Spanish," and the other participant said that they "ask and answer simple questions, when I have to expand, I get more nervous." All of the comments from the online interpersonal task mentioned forgetting words due to nervousness. For example, a participant got "very nervous because I forget simple phrases and vocabulary."

The similarity of the comments between in-class and online tasks regarding communication apprehension shows that the medium in which the oral task is complete does not seem to be a relevant factor. Considering forgetting information is a part of communication apprehension, these results are in accordance with Q5, "I feel so anxious that they forget information while completing oral tasks in class/online" in which there were only small difference between the answers.

\section{Preparedness}

Preparedness encompassed linguistic knowledge, pronunciation, learning formulaic structures, and work done outside of class. The inability to complete a task or the lack of understanding of task content and/or procedure may lead to experiencing higher levels of anxiety. Familiarity with the task is also related to preparedness since the variable of novelty of task is reduced. In this study, the oral tasks selected resemble many of the oral activities students do in the classroom in order to help decrease anxiety due to preparedness. . . Although students did not directly address the issue of familiarity with the task in their comments, results from Q4 from the survey showed that anxiety for online tasks (65\%) is higher than for in-class tasks (42\%).

In the surveys, the issue of preparedness was directly related to lack of linguistic knowledge. In the in-class presentational oral task, one participant said they were nervous because 
of "new vocab" while for another participant the "new words" made them feel uncomfortable. One participant said they felt "a little nervous because there was so much information." Considerably so, unfamiliarity with the vocabulary was a major theme as the participants learned they should have read over the new vocabulary the day before and then practiced it for the first time before completing the oral task. One positive feature of preparedness was that a participant felt the task was "easy for most part using vocabulary I know." Using known vocabulary may relate to Q4 about completing similar tasks. As repeated tasks give smore familiarity with the vocabulary, the lower anxiety for in-class tasks may come from this reinforcement of lexical items.

Similar to the in-class presentational oral task, a source of anxiety came from not understanding the vocabulary. For example, some comments were, “I wasn't understanding what I was asking," and "nervous because I am not sure what all the words meant." However, when asking questions, a positive aspect of preparedness was the ability to prepare what participants wanted to say. Some examples of this were, "comfortable because I could prepare my sentences" and "I felt more confident when I was given the words because it was a safety net." Another source of anxiety comes from teacher's feedback on students' questions. For example, a student worries about "not knowing what my teacher is saying back to me." Finally, task complexity came into play when looking at one positive comment. The participant said they felt "confident" because everything was "easy to explain."

The online interpersonal oral task also received mixed positive and negative comments One part of a student's frustration with the task came from their partner's linguistic abilities rather than their own. In regard to their partner, they said it was "hard to speak with them because they didn't know certain things." Also relating to Q4, another participant felt that novelty was a crucial factor into their anxiety saying that "I am not prepared for the orals when it is not what I expected." 
Finally, there was one positive comment about how the online oral tasks are completed. The participant mentioned "I feel like we have many resources to help us feel fully prepared for the oral tasks." As for preparedness and the medium through which the task is completed, there does not seem to be a difference as each participant mentioned having more time to learn lexical items or having more practice.

\section{Summary of results}

When comparing online and in-class tasks, online presentational tasks seem to trigger more FLA, but this anxiety does not seem to be manifested in physical reactions such as trembling. In addition, these online presentational tasks do not raise fear of negative evaluation or different manifestations of communication apprehension. Unfortunately, the results for test anxiety are unclear as online presentational oral tasks scored higher in both feeling anxious and not feeling anxious when it comes to making mistakes. However, participants only commented that they felt better during in-class tasks because they are not being graded.

As for in-class and online interpersonal tasks, the results are consistently close with one another when it comes to levels of anxiety. Online interpersonal tasks showed a higher level of anxiety associated with it for general anxiety and test anxiety. Online tasks had lower anxiety levels when it came to fear of negative evaluation and communication apprehension.

Overall, no clear differences based on medium appear when looking at different components of FLA such as trembling, fear of negative evaluation, test anxiety and forgetfulness. Results from the survey are relatively close between mediums. However, in general, participants reported higher anxiety while completing online tasks. 


\section{CHAPTER V: DISCUSSION}

This section sets out to examine and interpret the data collected and presented in the previous section in conjunction with comparing it to other studies to better understand and analyze the results. Through this, this discussion hopes to propose some new information about anxiety in beginning level language learners and how the use of CALL approaches affects these students. In order to do so, this section is separated into discussing the proposed research questions:

1. How do participants' levels of FLA differ between presentational oral tasks completed in the classroom and presentational oral tasks completed in an online interface?

2. How do participants' levels of FLA differ between interpersonal oral tasks completed in the classroom and interpersonal oral tasks completed in an online interface?

3. What factors are associated with different levels of FLA in oral tasks completed in the classroom and in an online interface?

\section{How do participants' levels of FLA differ between presentational oral tasks completed in the classroom and presentational tasks completed in an online interface?}

As far as this researcher knows, there is little work done on FLA and presentational oral tasks, as much research done focuses on interpersonal oral and written conversations. Therefore, there is no significant body of work to compare these results and the results for presentational tasks have been compared to anxiety measured in interpersonal tasks. The results of the current study investigate levels of FLA in two different mediums of presentational tasks, online and in-class, and will be compared to studies that have investigated anxiety in $\mathrm{CMC}$ and traditional classrooms though those studies have focused on text-based $\mathrm{CMC}$ and oral modes of $\mathrm{CMC}$ during interpersonal tasks. 
As for FLA in presentational tasks, the reported levels of anxiety indicate that participants feel more nervous during the online oral tasks in general. This is incongruous with typical research done in CMC as typically tasks online have resulted in lower levels of anxiety (Arnold, 2007; Choy \& Carey, 2001; Cote \& Gaffney 2018; Melchour-Couto 2017, 2018; Poza 2011; Warshauer, 1996). In our study, levels of anxiety in-class lowered when participants were asked to reflect on the familiarity of the task while levels of anxiety from online tasks stayed the same under the same circumstances. Therefore, completing tasks that are familiar to students does not impact on FLA during presentational oral tasks online.

However, before concluding that the online interface is solely responsible for higher anxiety levels, it is important to look at the online tasks in more detail. All online tasks were graded and therefore, test anxiety could be the reason for the results observed. In comparison, the in-class oral tasks were not graded on performance, but rather they were just another task in a series of tasks that they completed in the classroom. One participant commented on this difference when thinking about the presentational oral task saying that "this was not being graded so I did not mind messing up." Another reason for this increased anxiety can be that the online tasks were also timed whereas the in-class tasks had a more flexible time limit. Baralt and Gurzynski-Weiss (2011) also reported findings that their participants took twice as long to complete the CMC text-based chat as compared to a face-to-face task. Participants in the current study had two minutes to record their answer online although the activity was 5 minutes long whereas in the in-class task every participant spoke for about two minutes, but possibly had more time to think and also listen to other people's speeches.

Additionally, when students were asked to reflect on the fact that these tasks should be familiar to them from previous class work, we also observed that levels of anxiety raised under 
online conditions. However, in the in-class presentational task, the familiarity of the task seemed to help reduce anxiety. Interestingly enough, students commented on the helpfulness of completing similar tasks in the classroom as to those online, although they clearly indicated more anxiety in the online setting in their survey responses. Nonetheless, familiarity with the ask is an indicator of lower anxiety as evidenced in McBain et al.'s (2016) study where they noted that the lack of familiarity with the task can cause an increase in anxiety. However, the fact that the online tasks were graded and timed is most likely the main factor in interpreting these results because although the familiarity of the task impacted anxiety levels slightly, it was not enough to overcome that fear of being tested while completing the online oral tasks.

Fear of negative evaluation is one of the strongest indicators of anxiety but at the same time, it is the most positively affected by FLA reducing strategies (Alrabai, 2015). As expected, the in-class task triggered higher levels of anxiety related to peer comparison since students had to present their families to each other in small groups. The lack of audience and interaction during the online presentational task is responsible for a lower anxiety level related to fear of negative evaluation by their peers. One participant mentioned that they "feel more comfortable with the oral tasks online. These make me feel less pressure as it's only me and a computer." This is congruent with findings from Warshauer (1996) who explains that without paralinguistic cues, such as looks of confusion or body language, anxiety is lower.

A similar result was found in Poza's (2011) study where participants had a conversation with a partner through asynchronous oral recordings. Another comparable finding was also reported by Melchor-Couto (2017) with his participants in the Second Life platform where the anonymity of their avatars helped students feel more comfortable. Overall, the majority of students in our study did not fear being compared with their peers, but the online tasks resulted in the lowest 
level of anxiety even though students knew the instructor would listen to their performance and grade it. It looks like test anxiety did not play a role here and students seem more fearful of peercomparison when speaking with each other.

As mentioned, the main difference between the online and the in-class presentational task was the presence or absence of an audience. While performing the in-class tasks, participants were in small groups with their peers while when performing online tasks even though all participants were in a computer lab, each person was focusing on their own work. Results from worrying about others speaking better Spanish show that online tasks had lower anxiety relating to fear of negative evaluation (Online: 17\%, In-Class: 30.7\%). Baralt and Gurzynski-Weiss (2011) also found higher anxiety relating to fear of negative evaluation in face-to-face tasks as students feel they notice their mistakes more due to looks from the listeners.

Another noticeable difference between the in-class and the online presentational oral task is that when it came to physical reactions due to FLA overall this physical expression of anxiety was not a trait that many of the participants shared; however, some differences exist based on the medium of the task. None of the participants reported that they tremble when they are completing an online oral task online while $23 \%$ of participants in the in-class oral task said that they did. This lower FLA in the online presentational oral task differs from results obtained by Baralt and Guzynski (2011), who reported that although there were slight differences between CMC and face to face tasks, there was no significant difference in FLA between CMC and face to face tasks .

One participant echoed a reason as to this difference too saying that they feel confident when they "ask and answer simple questions." The presentation of the family tree in front of their small groups of peers may also result in some students asking for clarification or asking a followup question. The ability to ask and answer questions of the presenter may have influenced anxiety 
during the in-class task which could explain a higher level of trembling. The online presentational task was a task the participants had to narrate their daily routines and therefore did not have to elaborate more than was necessary for the time limit. In addition, in the classroom, many activities include answering questions orally, so familiarity with the task can also explain why students were not trembling as much when doing the online presentational task.

In conclusion, levels of FLA in in-class presentational tasks are lower than those of online tasks when looking at anxiety in general. Comments have suggested that FLA in relationship to test anxiety is also lower in in-class tasks but due to the constraints of the current study that is inconclusive. However, FLA in online tasks are lower than those of in-class tasks when examining fear of negative evaluation and physical responses. Reasons for this are that during online tasks, there is no audience listening to the speaker therefore paralinguistic cues that tells the speaker that they are making mistakes.

\section{How do participants' levels of FLA differ between interpersonal oral tasks completed in the classroom and interpersonal oral tasks completed in an online interface?}

As with presentational oral tasks, not much research has been done with audio and video chats in regard to FLA. In regard to interpersonal tasks, much of the research in CMC has looked at text-based CMC and FLA (Arnold, 2007; Baralt \& Gurzynski, 2011; Côte and Gaffney, 2018 Warshauer, 1996). Nonetheless, some studies (Cho \& Carey, 2001; Poza, 2011) have looked at asynchronous oral chats through the use of a program called Wimba to see how FLA is experienced through CALL approaches and Melchor-Couto $(2017,2018)$ looked at how virtual worlds could help reduce FLA in foreign language students.

In the same way as with the presentational task, during interpersonal tasks the online interface intensified participants' level of anxiety, even when they knew the task was familiar to 
them. This result suggests that task type is not one of the factors that impact anxiety. Task type becomes a relevant factor during interpersonal tasks when we look at participants' fear of making mistakes and their physical reaction of trembling caused by anxiety. In both cases the in-class interpersonal tasks resulted in higher levels of anxiety. Our results are not supported by Baralt and Gurzynski-Weiss' (2011) findings where they did not find a significant difference between anxiety in face-to-face conversations as compared to CMC during interpersonal oral tasks. Due to the small number of participants in the current study, we were not able to conduct statistical analysis, and therefore, our findings need to be taken with caution.

However, Baralt and Gurzynski (2011) also explain that novelty of using technology and time on task were factors that contributed to anxiety. Baralt and Gurzynski's study (2011) was published nine years prior to the current study, but since then, technology has noticeably evolved and most importantly, university students today are very familiar with technological tools. Therefore, the novelty factor mentioned in Baralt and Gurzynski's study (2011) cannot help us explain our findings.

In regard to fear of negative evaluation, online oral tasks rated lower in anxiety than the inclass oral tasks. One notable factor about this difference is that the anxiety in in-class interpersonal oral tasks were rated more severely than the online counterpart. In Melchour-Couto's (2017) study researchers looked at how using virtual classrooms could reduce FLA in students, and they found that participants were gaining more self-confidence and becoming more comfortable as the weeks went by. As the online activities for this current study took place after the in-class activities, participants might have felt more comfortable with their online partner than the constant changing of partners in the classroom. 
However, Melchour-Couto (2017) also found that when looking at spontaneous interpersonal speech, fear of negative evaluation had a slight increase online, but in this current study fear of negative evaluation was rated slightly lower in the online task than in the in-class interpersonal oral task. As Melchour-Cuoto (2017) explains not being physically present make participants feel at ease. Even though our participants completed the oral task in a language lab, they were separated by some degree from their partner. The results of the present study more closely relate to Poza's (2011) findings that participants completing the face-to-face and the asynchronous oral chat had similar anxiety levels, but the asynchronous group had a greater number of participants with lower anxiety as compared to high.

The findings on test anxiety and making mistakes from this present study show that online interpersonal tasks had a higher anxiety score. This finding agrees with Poza (2011) who stated in their study on asynchronous interpersonal oral chats that the participants are more worried about making mistakes online. They attributed this to the fact that participants were able to modify and edit their responses as they were recording their answers. However, in the present study, the participants were being recorded live and could not redo their answers. Poza (2011) attributed having more time to rerecord an audio if the speaker was unhappy with their performance to higher anxiety levels about making mistakes. However, the current study did not afford participants to edit or rerecord their online interpersonal task. Having the ability, or not, to correct an error may not affect anxiety in oral tasks differently.

Cote and Gaffney (2018), in researching synchronous text-based CMC, found opposite results and reported that anxiety was lower in synchronous text-based CMC than in face-to-face interpersonal tasks. They purported two reasons for lower anxiety, (1) a lack of being face-to-face, and (2) having more time to edit and plan output. As for not being face-to-face, the current study 
found that there was lower reported anxiety in fear of negative evaluation in online tasks, which may be in agreement with Cote and Gaffney's reasoning. There were no comments made about feeling negatively evaluated by their partner in the online oral task, while many comments were made on that topic for the in-class oral task. As for time, although Cote and Gaffney (2018) and the current study both look at synchronous conversations, text-based chats seem to afford the interlocutors more time to be able to process input and plan output while synchronous audio chats have a certain amount of urgency that is similar to face-to-face conversations.

Overall, this study has found that interpersonal oral tasks completed online have higher levels of general anxiety, but lower anxiety levels in fear of negative evaluation and communication apprehension. Fear of negative evaluation may be lower in online tasks, as theorized by Cote and Gaffney (2018), because the students are simply not face-to-face. A large portion of comments described feeling discomfort and anxious because of the way their speaking partner and classmates were looking at the speaker during interpersonal in-class tasks.

Time as also a factor in the current study that raised reported anxiety, however it seems that having time to process may also reduce anxiety. Having an urgency to answer questions quickly and think of a response can cause anxiety. As mentioned above, having time to process and create output helps lower anxiety in students (Cote \& Gaffney, 2018), however having the opportunity to try to perfect the output may increase anxiety (Poza, 2011). This could be attributed to the fact that anxiety has some related features to perfectionism (Gregersen \& Horwitz, 2002), and therefore, having more time might allow students to focus too much on error correction.

Our interpersonal tasks involved synchronous oral speech, since interlocutors are speaking at the same time. Therefore, the online oral tasks are very similar to in-class pair activities where there is an immediate need to receive input, process it, and produce intelligible output in a 
relatively quick manner. The similarity between online and in-class in this regard is shown by the closeness of levels of anxiety reported among the FLA survey in which only trembling had a difference larger than $10 \%$.

\section{What factors are associated with different levels of FLA in oral tasks completed in the}

\section{classroom and in an online interface?}

Looking at the qualitative data, we can see that the biggest comments about the oral tasks were fear of negative evaluation, test anxiety, communication apprehension and general preparedness. When analyzing what anxiety lowering approaches either the instructor or student could have used, the most commented items were examples on how to complete the activity, scaffolding activities, clear instructors on how to complete the activity and changing time limitations. The participants commented that the item they could do the most to help lower their anxiety was to study or speak more.

When looking at fear of negative evaluation in online and in-class tasks, one positive aspect was that many participants felt that knowing the class and feeling comfortable with their classmates helped them feel more comfortable when having to do in-class tasks. Seeing peers also struggle with tasks helped lower anxiety as well. This correlates with Melchor-Couto (2018) comments that anonymity is not a factor in decreasing anxiety in long term pairing because the interlocutors get to know each other from their multiple conversations. However, other studies have shown that anonymity helps reduce anxiety (Arnold, 2007; Melchor-Couto, 2017), which may actually be related to not seeing their partner rather than not knowing who they are as we believe helped lower anxiety.

The time allotted to complete oral tasks online is a factor that contributes to higher levels of FLA, similarly to what other research has revealed (Baralt \& Gurzynski-Weiss, 2011; Cote \& 
Gaffney, 2018; Poza, 2011). The time limit may have rushed participants in the current study to finish the online tasks resulting in higher anxiety levels. Baralt and Gurzynksi-Weiss (2011) found that having extra time for online and in-class tasks lowers anxiety.

Nonetheless, it is important to note that much of the research in FLA does not focus on synchronous tasks (Baralt \& Gurzynski-Weiss, 2011; Poza, 2011) or the tasks were synchronous but communication was text-based (Cote and Gaffney,2018). The agency in synchronous oral chatting does not allow for large gaps of time to pass between questions and answers. The time limit on the online oral chats in the current study were designed to promote more spontaneous chatting and less practiced and rehearsed oral performance although ultimately this time limit may have caused higher anxiety levels.

Finally, as in McBain et al.'s (2016) study, a low percent of students experienced anxiety due to the task at hand while other students reported anxiety due to not understanding the task. The present study also found that there is a need for clear and concise instructions and modeling of the activity in order for the students to understand what is expected of them. Having unclear expectations of the outcome is another factor that can raise anxiety.

In general, the factors that induced anxiety in the participants were all commonly mentioned FLA concepts such as test anxiety, communication apprehension and fear of negative evaluation. The novelty of the task and having clear instructions and expectations is also a large factor in how students feel when they need to complete tasks. These factors were consistent among the in-class and online oral activities and there was no difference between them when looking at why the participants felt anxious. 


\section{CHAPTER VI: CONCLUSION}

This study was designed with the objective to contribute to the previous literature on FLA and CMC and CALL approaches. Some literature has revealed interesting findings about FLA (Arnold, 2007; Baralt \& Gurzynski-Weiss, 2011; Cho \& Carey, 2001; Cote \& Gaffney, 2018; Melchour-Couto, 2017, 2018; Poza, 2011) but not much research exists on the use of synchronous audio and video CMC impact FLA. This type of research is necessary considering the rise of new asynchronous and synchronous technology in education, such as Flipgrid, Zoom and YouTube-

After reviewing and discussing the data presented in this study, online CMC tasks indicated a raise in anxiety. When students are asked about feeling anxious in online and in-class oral tasks, they report that online oral tasks raise anxiety overall. However, it can be seen that audio-based CMC results in lower levels of anxiety when it comes to fear of negative evaluation. This has been studied before by Melchour-Couto $(2017,2018)$ but through the lens of a virtual world where the participants did not know each other while this current study introduced the use of CMC in a blended classroom in which participants used technology to complete oral tasks outside of the class period. Finally, although this study did not look specifically at task type, comparing the data between presentational and interpersonal tasks, task type did not show large differences when it came to FLA.

Out findings also indicate that in order to lower anxiety in participants, they need to feel confident about the oral task through explicit clear tasks instructions, use of models and clear task outcome expectations. Although this study used a time limit for the online tasks in order to have participants produce spontaneous speech without preparing ahead of time, having either a visible time limit, or having a time limit increased anxiety for our participants. Another anxiety reducing 
strategy is to have a positive classroom environment in which students can speak freely without feeling judged by either their instructor or their peers.

One pedagogical implication that this study has revealed, in accordance with previous studies on CALL approaches, is that there are benefits to using CMC. Synchronous audio-based CMC resembles face-to-face conversations because of the immediacy of the communicative act. Participants need to process input and offer output in real time. The benefits of engaging in synchronous audio-based tasks, as the ones in this study, include pronunciation practice, something participants comments on in the survey and the opportunity to build confidence to speak and make mistakes. In class, students are often placed in pairs to conduct interpersonal oral tasks, but their output is often assessed by the teacher which increases FLA. During online interpersonal oral tasks, students seem more concerned about getting their message across and therefore, they are not so focused being evaluated by their partner when they make a mistake.

As with every study, this current study is not without limitations. When reporting on FLA immediately after the oral task, participants were prompted to think about the last task completed during the class time. However, due to the manner in which the survey questions were written, it can be interpreted that participants responded about in-class tasks and online-tasks in general. Therefore, the interpretations of the data with regard to task type need to be taken with caution. Another factor to consider is that the time between the oral task in class and the oral task online was over two weeks and participants might have gotten more comfortable speaking Spanish as they have had more practice. As previously mentioned, the online tasks were graded while the inclass oral tasks were not, which could have influenced the results in all areas as evidenced by some of the answers to the open-ended questions from participants. Given that Baralt and GurzynskiWeiss (2011) found that the length of time involved in the task could influence anxiety, time is 
another variable that needs to be considered when looking at these tasks as the in-class oral activity could allow participants more time and more flexibility to talk and figure out what they want to say. Additionally, in the in-class task many learners listened to their partners speak first, which becomes a model that the students could use when their turn to speak would come. The online task, on the other hand, had the added time pressure as students would see the physical timer in front of them counting down. Finally, all the data collected on anxiety was self-reported, which means that each participant may have a different understanding of what they felt or may not have wanted to report their anxiety.

Given the limitations of this study, future research should consider assessing participants' FLA before participating in the study in order to see how their anxiety change between the different types of oral tasks and the medium. Additionally, in examining FLA and presentational oral tasks, one could use virtual reality technology that allows users to feel like they are giving a presentation in front of an audience to see how students react to a simulated audience. This could also be done by setting up groups in a videochat program such as Zoom or Skype and having participants complete presentational oral tasks in a group videochat. Investigating FLA in video-CMC as compared to audio-CMC, text-based CMC and face-to-face would be another innovative way to see how students interact through online mediums.

Finally, even though this current study used open-ended questions after the abbreviated FLACS survey, the data given could not be thoroughly examined, and some questions were left unanswered to the researcher. A study in which participants have an open-ended interview with the researchers after completing the oral tasks would be beneficial in order to understand how students think when they are completing oral tasks online and in the classroom in order to use this data to better triangulate results. Finally, seeing how students communicate through video CMC 
and audio CMC would be another interesting study in order to examine the relationship between FLA and the intricacies of personal interaction such as how students negotiate meaning, interact and do error correction. 


\section{REFERENCES}

Abrams, Z. I. (2003). The effect of synchronous and asynchronous CMC on oral performance in German. The Modern Language Journal, 87(2), 157-167.

Absalom, M., \& Pais Marden, M. (2004). Email communication and language learning at university-An Australian case study. Computer Assisted Language Learning, 17(3-4), 403-440.

Alrabai, F. (2015). The influence of teachers' anxiety-reducing strategies on learners' foreign language anxiety. Innovation in Language Learning and Teaching, 9(2), 163-190.

Arnaiz, P., \& Guillén, P. (2012). Foreign language anxiety in a Spanish university setting: Interpersonal differences. Revista de Psicodidáctica, 17(1), 5-26.

Arnold, J., \& Brown, H. D. (1999). A map of the terrain. In J. Arnold (Ed.), Affect in language learning. Cambridge: Cambridge University Press.

Arnold, N. 2007. Reducing foreign language communication apprehension with computermediated communication: a preliminary study. System 35, no. 4: 469-486.

Backman, N. (1976). Two measures of affective factors as they relate to progress in adult second-language learning. Ontario Institute for Studies in Education.

Baralt, M. and L. Gurzynski-Weiss. 2011. Comparing learners' state anxiety during task-based interaction in computer- mediated and face-to-face communication. Language Teaching Research 15, no. 2: 201-229.

Blake, R., Wilson, N. L., Cetto, M., \& Pardo-Ballester, C. (2008). Measuring oral proficiency in distance, face-to-face, and blended classrooms. Language Learning \& Technology, 12(3), 114. 
Boudreau, C., MacIntyre, P., \& Dewaele, J. M. (2018). Enjoyment and anxiety in second language communication: An idiodynamic approach. Studies in Second Language Learning and Teaching, 8(1), 149-170.

Brandl, K. (2008). Communicative language teaching in action: Putting principles to work. Pearson/Prentice Hall.

Brooks, L. (2009). Interacting in pairs in a test of oral proficiency: Co-constructing a better performance. Language testing, 26(3), 341-366.

Campbell, C. M., \& Shaw, V. M. (1994). Language anxiety and gender differences in adult second language learners: Exploring the relationship. In C. A. Klee (Ed.), Faces in a crowd: The individual learner in multisection courses (pp. 47-80). Boston: Heinle \& Heinle.

Chapelle, C. A. (2009). The relationship between second language acquisition theory and computer-assisted language learning. The modern language journal, 93, 741-753.

Chastain, K. (1975). Affective and ability factors in second-language acquisition. Language learning, 25(1), 153-161.

Cheng, Y. (2002) Factors associated with foreign language writing anxiety. Foreign Language Annals, 35(6): 647-656.

Cho, S. P., \& Carey, S. (2001). "Increasing Korean oral fluency using an electronic bulletin board and Wimba-based voiced chat." The Korean Language in America, 6, 115-128.

Clément, R., Gardner, R. C., \& Smythe, P. C. (1980). Social and individual factors in second language acquisition. Canadian Journal of Behavioural Science/Revue canadienne des sciences du comportement, 12(4), 293. 
Côté, S., \& Gaffney, C. (2018). The effect of synchronous computer-mediated communication on beginner L2 learners' foreign language anxiety and participation. The Language Learning Journal, 1-12.

Daly, J. (1991). Understanding communication apprehension: An introduction for language educators. Language anxiety: From theory and research to classroom implications, 9(1), $3-13$.

Daly, J. A., \& Shamo, W. (1977). Academic decisions as a function of written and oral communication apprehension. Annual Convention of the International Communication Association, Berlin.

De Los Arcos, B., Coleman, J. A., \& Hampel, R. (2009). Learners' anxiety in audiographic conferences: a discursive psychology approach to emotion talk. ReCALL, 21(1), 3-17.

Dewaele, J. M. (2002). Psychological and sociodemographic correlates of communicative anxiety in L2 and L3 production. International Journal of Bilingualism, 6(1), 23-38.

Dewaele, J.-M. 2007. Predicting language learners' grades in the L1, L2, L3, and L4: the effect of some psychological and sociocognitive variables. International Journal of Multilingualism 4: 169-197.

Dewaele, J. M., MacIntyre, P. D., Boudreau, C., \& Dewaele, L. (2016). Do girls have all the fun? Anxiety and enjoyment in the foreign language classroom. Theory and practice of second language acquisition, 2(1), 41-63.

Dewaele, J. M., Petrides, K. V., \& Furnham, A. (2008). Effects of trait emotional intelligence and sociobiographical variables on communicative anxiety and foreign language anxiety among adult multilinguals: A review and empirical investigation. Language learning, 58(4), 911-960. 
Donovan, L. A., \& MacIntyre, P. D. (2005). Age and sex differences in willingness to communicate, communication apprehension, and self-perceived competence. Communication Research Reports, 21(4), 420-427.

Dörnyei, Z. (2009). The psychology of second language acquisition. Oxford: Oxford University Press.

Elkhafaifi, H. (2005). Listening comprehension and anxiety in the Arabic language classroom. The Modern Language Journal 89.2, 206-220.

Ellis, R. (1994). The Study of Second Language Acquisition. New York: Oxford University Press.

Gardner, R. C., \& Maclntyre, P. D. (1991). An instrumental motivation in language study: Who says it isn't effective? Studies in Second Language Acquisition, 13, 57-72.

Gardner, R. C., \& MacIntyre, P. D. (1993). On the measurement of affective variables in second language learning. Language learning, 43(2), 157-194.

Gardner, R. C., Smythe, P. C., \& Brunet, G. R. (1977). Intensive second language study: Effects on attitudes, motivation and French achievement. Language Learning, 27, 243-261.

Gardner, R. C., Clément, R., Smythe, P. C., \& Smythe, C. C. (1979). Attitudes and motivation test battery, Revised manual (Research Bulletin No. 15). London: Unversity of Western Ontario.

Gardner, R. C., Smythe, P. C., Clément, R., \& Gliksman, L. (1976) Second language Learning: A sociological and psychological perspective. Canadian Modern Language Review, 32, $198-213$. 
Gardner, R. C., Smythe, P. C., \& Lalonde, R. N. (1984) The nature an replicability of factors in second language acquisition (Research Bulletin No. 605). London: University of Western Ontario.

Gregersen, T.S. (2003), To Err Is Human: A Reminder to Teachers of Language-Anxious Students. Foreign Language Annals, 36: 25-32.

Gregersen, T. and E.K. Horwitz. 2002. Language learning and perfectionism: anxious and nonanxious language learners' reactions to their own oral performance. The Modern Language Journal 86, no. 4: 562-570.

Hampel, R. (2003) Theoretical perspectives and new practices in audio-graphic conferencing for language learning. ReCALL, 15(1): 21-35.

Hampel, R. (2006). Rethinking task design for the digital age: A framework for language teaching and learning in a synchronous online environment. ReCALL, 18(1), 105-121.

Hilgard, E., Atkinson, R. C., Atkinson, R. L., \& Lambert, W. W. (1971). Introduction to Psychology. Hardcourt Brace Jovanovich.

Holbrook, H. T. (1987). Communication Apprehension: The Quiet Student in Your Classroom. ERIC Digest.

Horwitz, M. B. (2002). Communication Apprehension. Albany, NY: Delmar Singular

Horwitz, E. (1986). "Preliminary evidence for the reliability and validity of a foreign language anxiety scale.” TESOL Quarterly, 20, 559-562.

Horwitz, E. (2001). Language anxiety and achievement. Annual review of applied linguistics, 21, $112-126$.

Horwitz, E. K., \& Young, D. J. (1991). Language anxiety: From theory and research to classroom implications. Pearson College Div. 
Kern, R. 1995. Restructuring classroom interaction with networked computers: effects on quantity and characteristics of language production. The Modern Language Journal 79: $457-476$.

Kitano, K. (2001) Anxiety in the college Japanese language classroom. The Modern Language Journal, 85(4): 549-566.

Kleinmann, H. H. (1977). Avoidance behavior in adult second language acquisition. Language Learning, 27, 93-107.

Lamendella, J. T. (1977). The limbic system in human communication. In Studies in neurolinguistics (pp. 157-222). Academic Press.

Levelt, W. J. M. (1989). Speaking: From intention to articulation. Cambridge, MA: The MIT Press.

Lewis, M. D. (2005). Bridging emotion theory and neurobiology through dynamic systems modeling. Behavioral and brain sciences, 28(2), 169-194.

Long, M. H. (1989). Task, group, and task-group interactions. University of Hawaii's Working Papers in ESL, 8(2), 1-26.

Long, M. (2015). TBLT: Building the road as we travel. In Domains and Directions in the Development of TBLT (pp. 1-26). John Benjamins.

Koba, N., Ogawa, N., \& Wilkinson, D. (2000). Using the community language learning approach to cope with language anxiety. The Internet TESL Journal, 6(11), 1-5.

Macher, D., Paechter, M., Papousek, I., Ruggeri, K., Freudenthaler, H. H., \& Arendasy, M. (2013). Statistics anxiety, state anxiety during an examination, and academic achievement. British Journal of Educational Psychology, 83(4), 535-549. 
Ohman, A. (2000). "Fear and anxiety: Evolutionary cognitive, and clinical perspectives". In M. Lewis \& J. M. Haviland-Jones (Eds.), Handbook of Emotions, (pp.573- 593). New York: The Guilford Press.

MacIntyre, P. D. (1995). How does anxiety affect second language learning? A reply to Sparks and Ganschow. The modern language journal, 79(1), 90-99.

MacIntyre, P. D. (1999). Language anxiety: A review of the research for language teachers. Affect in foreign language and second language learning: A practical guide to creating a low-anxiety classroom atmosphere, 24, 41.

MacIntyre, P. D. (2002). Motivation, anxiety and emotion in second language acquisition. Individual differences and instructed language learning, 2, 45-68.

MacIntyre, P. D. (2012). The idiodynamic method: A closer look at the dynamics of communication traits. Communication Research Reports, 29(4), 361-367.

MacIntyre, P. D., \& Gardner, R. C. (1989). Anxiety and second-language learning: Toward a theoretical clarification. Language learning, 39(2), 251-275.

MacIntyre, P. D., \& Gardner, R. C. (1991a). Language anxiety: Its relationship to other anxieties and to processing in native and second languages. Language learning, 41(4), 513-534.

MacIntyre, P. D., \& Gardner, R. C. (1991b). "Methods and results in the study of anxiety and language learning: A review of the literature.’Language Learning, 41, 85-117.

MacIntyre, P. D., \& Gardner, R. C. (1994). The subtle effects of language anxiety on cognitive processing in the second language. Language learning, 44(2), 283-305. 
McBain, B., Drew, A., James, C., Phelan, L., Harris, K. M., \& Archer, J. (2016). Student experience of oral communication assessment tasks online from a multi-disciplinary trial. Education+ Training.

McCroskey, J. C. (1984). The communication apprehension perspective. Avoiding communication: Shyness, reticence, and communication apprehension, 13-38.

Melchor-Couto, S. (2017). Foreign language anxiety levels in Second Life oral interaction. ReCALL, 29(1), 99-119.

Melchor-Couto, S. (2018). Virtual world anonymity and foreign language oral interaction. $\operatorname{ReCALL,30(2),~232-249.~}$

Norton, A. R., \& Abbott, M. J. (2016). Self-focused cognition in social anxiety: A review of the theoretical and empirical literature. Behaviour Change, 33(1), 44-64.

Onwuegbuzie, A., Bailey, P., \& Daley, C. (1999). Factors associated with foreign language anxiety. Applied Psycholinguistics, 20(2), 217-239.

Onwuegbuzie, A. J., Bailey, P., \& Daley, C. E. (2000). “Cognitive, affective, personality, and demographic predictors of foreign language achievement." Journal of Educational Research, 94, 3-16.

Onwuegbuzie, A. J., Bailey, P., \& Daley, C. E. (2002). The role of foreign language anxiety and students' expectations in foreign language learning. Research in the Schools, 9(1), 33-50.

Park, H., \& Lee, A. R. (2005). L2 learners' anxiety, self-confidence and oral performance. In Proceedings of the 10th Conference of Pan-Pacific Association of Applied Linguistics(pp. 107-208).

Payne, S., \& Whitney, P. J.(2002). Developing L2 oral proficiency through synchronous CMC: Output, working memory, and interlanguage development. 
Pelletieri, J. (2000). "Negotiation in cyberspace: The role of chatting in the development of grammatical competence.”M. Warschauer \& Kern, R. G. (Eds.), Network-Based Language Teaching: Concepts and Practice (pp. 59-86). Cambridge: Cambridge University Press.

Poza, M. I. C. (2011). The effects of asynchronous computer voice conferencing on L2 learners' speaking anxiety. IALLT Journal of Language Learning Technologies, 41(1), 33-63.

Price, M. L. (1991). The subjective experience of foreign language anxiety: Interviews with highly anxious students. In Horwitz \& Young (eds.), 101-108.

Richmond, V. P. (1984). Implications of quietness: Some facts and speculations. Avoiding communication: Shyness, reticence, and communication apprehension, 145-155.

Richmond, V. P., \& McCroskey, J. C. (1988). Communication: Apprehension, avoidance, and effectiveness. Scottsdale, AZ: Gorsuch Scarisbrick.

Sanchez-Herrero, S. A., \& Sanchez, M. D. P. (1992). The Predicitive Validation of an Instrument Designed to Measure Student Anxiety in Learning a Foreign Language. Educational and Psychological Measurement, 52(4), 961-966.

Schwartz, L. L. (1972). Educational psychology: Focus on the learner. Holbrook Press.

Scida, E. E., \& Jones, J. N. (2016). New tools, new designs: A study of a redesigned hybrid Spanish program. calico journal, 33(2), 174.

Scovel, T. (1978). The effect of affect on foreign language learning: A review of the anxiety research. Language learning, 28(1), 129-142.

Spielberger, C. (1966). The effects of anxiety on performance in complex learning tasks. Anxiety and behavior, 361-396. 
Spiegelberger, C. D. (1972). Anxiety: Current trends in theory and research. New York: Academic Press.

Swain, M. (1977). Future Directions in Second Language Research.

Swain, M., \& Burnaby, B. J. (1976). Personality Characteristics and Second-language Learning in Young Children: A Pilot Study. Ontario Institute for Studies in Education.

Taylor, L. (2000). Investigating the paired speaking test format. University of Cambridge ESOL Examinations Research Notes, 2, 14-15.

Tobias, S. (1986) Anxiety and cognitive processing of instruction. In R. Schwarzer (Ed.), Selfrelated cogitation in anxiety and motivation. Hillsdale, NJ: Erlbaum.

Tucker, G. R., Hamayan, E., \& Genesee, F. H. (1976). Affective, cognitive and social factors in second-language acquisition. Canadian modern language review, 32(3), 214-226.

Vogely, A. J. (1998). Listening comprehension anxiety: Students' reported sources and solutions. Foreign Language Annals, 31(1), 67-80.

Wang, C., \& Zhu, L. (2020, January). Effects of Content-Based Communicative Language Teaching in University English Writing Classes. In International Forum of Teaching \& Studies (Vol. 16, No. 1).

Ward, R., Moule, P., \& Lockyer, L. (2008). Adoption of Web 2.0 technologies in education for health professionals in the UK. Where are we and why?

Warschauer, M. (1996) Computer-mediated collaborative learning: Theory and practice. The Modern Language Journal, 81(4): 470-481.

Woodrow, L. J. (2006). “Anxiety and speaking English as a second language: English language speaking anxiety in a second language environment.” RELC Journal, 37, 308-328. 
Yan, J. X., \& Horwitz, E. K. (2008). Learners' perceptions of how anxiety interacts with personal and instructional factors to influence their achievement in English: A qualitative analysis of EFL learners in China. Language learning, 58(1), 151-183.

Young, D. J. (1991). Creating a low-anxiety classroom environment: What does the anxiety research suggest? The Modern Language Journal 75.4, 426-439.

Yu, W., She, H., \& Lee, Y. (2010). The effects of web-based/non-web-based problem solving instruction and high/low achievement on students' problem-solving ability and biology achievement. Innovations in Education and Teaching International, 47(2), 187-199.

Zayas-Bazán, E. (2005). Arriba!: comunicación y cultura. Prentice Hall. 


\section{APPENDIX A: STUDENT BACKGROUND INFORMATION}

Student Background Information

1. Name:

2. How do you prefer to be called, if different from above?:

3. Please, write down the number of Spanish/French/German courses you have taken in the past, when and where.

Language Level When Where

4. Why are you taking this course?

5. If you have not taken any foreign language course before, have you ever been exposed to a foreign language through friends, travel, music, etc.? Please, explain.

6. What are some of your personal interests? (tv shows you like to watch, what you like to do in your spare time, reading material you like, types of music, movies, sports, etc.....). This information will help your instructor prepare class activities close to students' interests.
a. TV shows:
b. Movies:
c. Hobbies:
d. Books:
e. Sports

f. Other interests:

7. If this is your first foreign language class at ISU, have you taken the online placement test (located at the department's website) to determine your level (111/112)?

1. YES NO

8. If not, how did you decide to register for this level 


\section{APPENDIX B: IN-CLASS PRESENTATIONAL TASK}

\section{Draw a small family tree labeling your family. Think about what they are like.}

Present your family to your fellow classmates. Explain:

the names of each person (Mi padre se llama Pol),

How many aunts/uncles/siblings/cousins (Tengo dos tías, se llaman Tina y Juaquína),

What each person does (Mi hermano trabaja en un banco)

Their favorite activities (Mi prima corre en el parque, a mi prima le gusta correr). 


\section{APPENDIX C: ONLINE PRESENTATIONAL ORAL TASKS}

You will be assessed on your use of grammar, vocabulary, and fluency. If you complete the assignment with appropriate grammar, vocabulary, and content, you will get a good score (80-90\%). You will get a higher score if you go beyond the exercise and add details and elaborate (90-100\%). Also, remember that if your instructor SUSPECTS you are reading something you wrote, he/she will ask you to complete a similar activity in his/her office.

1) Describe in detail your daily routine. Say at what time you do different things. Use verbs such as levantarse, acostarse, dormirse, etc. Speak between ONE and TWO minutes. 


\section{APPENDIX D: IN-CLASS INTERPERSONAL ORAL TASKS}

You need to plan a party with your friend. Fill in the missing information by asking questions to your partner with words such as ¿Quién? ¿Qué hace? ¿Cuándo? Y ¿Dónde?

\begin{tabular}{|l|l|}
\hline Invitar a & Nuestros amigos \\
\hline Llevar la música & Carlos \\
\hline & Roberto \\
\hline Levar las bebidas & Clara \\
\hline Llevar el altavoz & \\
\hline Limpiar & \\
\hline Hora (empezar) & \\
\hline Lugar (place) & En el parque \\
\hline El día & Viernes \\
\hline
\end{tabular}

Modelo: ¿A quienes invitamos a la fiesta?

Invitamos a nuestros amigos.

You need to plan a party with your friend. Fill in the missing information by asking questions to your partner with words such as ¿Quién? ¿Cuándo? Y ¿Dónde?

\begin{tabular}{|l|l|}
\hline Invitar a & Nuestros amigos \\
\hline Llevar la música & \\
\hline Llevar la comida & Roberto \\
\hline & Clara \\
\hline Llevar el altavoz & Marina \\
\hline Limpiar & YO \\
\hline Hora (empezar) & A las siete de la tarde \\
\hline Lugar (place) & \\
\hline El día & \\
\hline
\end{tabular}

Modelo: ¿A quienes invitamos a la fiesta?

Invitamos a nuestros amigos. 


\section{APPENDIX E: ONLINE INTERPERSONAL ORAL TASKS}

A

You and a friend just moved into an apartment with Juana, but you're not sure what she does during the day. Fill in the missing information by ASKING QUESTIONS to your partner about AT WHAT TIME Juana does her daily activities. Complete the task by filling in the time in each block.

Model: ¿A qué hora se despierta Juana?

Juana se despierta a las seis.

¿A qué hora Juana se duerme?

Juana se duerme a las seis y cuarto.

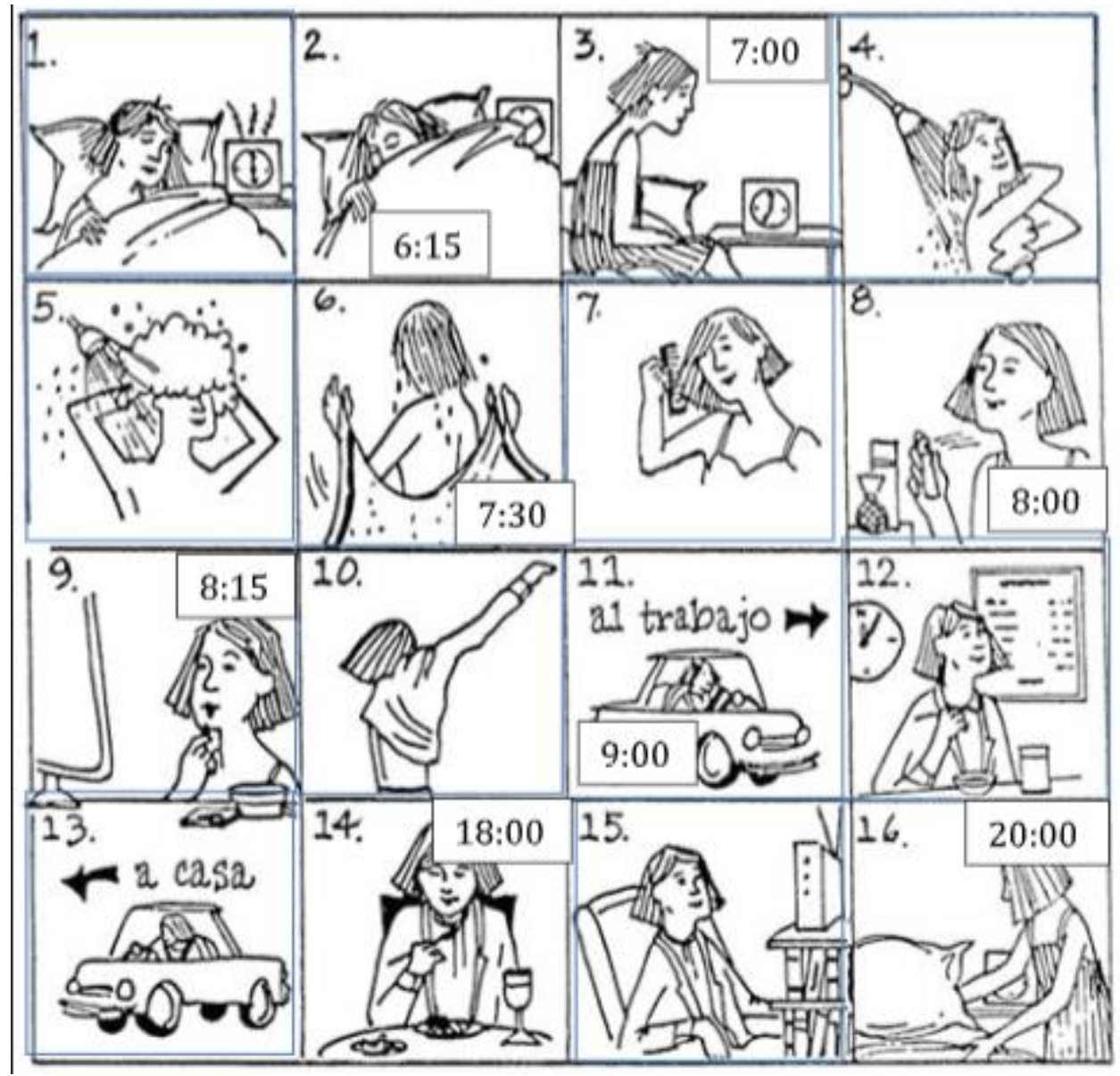


THIS TASK IS COMPLETED ONLY IN SPANISH. ASK YOUR PARTNER QUESTIONS IN SPANISH IF YOU DO NOT KNOW THE WORD.

¿Cómo se dice wake up? Se dice despertarse

¿Qué significa despertarse? Significa wake

up.

Repite por favor

¿Qué hace Juana en numero 4?

B

You and a friend just moved into an apartment with Juana, but you're not sure what she does during the day. Fill in the missing information by ASKING QUESTIONS to your partner about AT WHAT TIME Juana does her daily activities. Complete the task by filling in the time in each block.

Model: ¿A qué hora se despierta Juana?

Juana se despierta a las seis.

¿A qué hora Juana se duerme?

Juana se duerme a las seis y cuarto. 


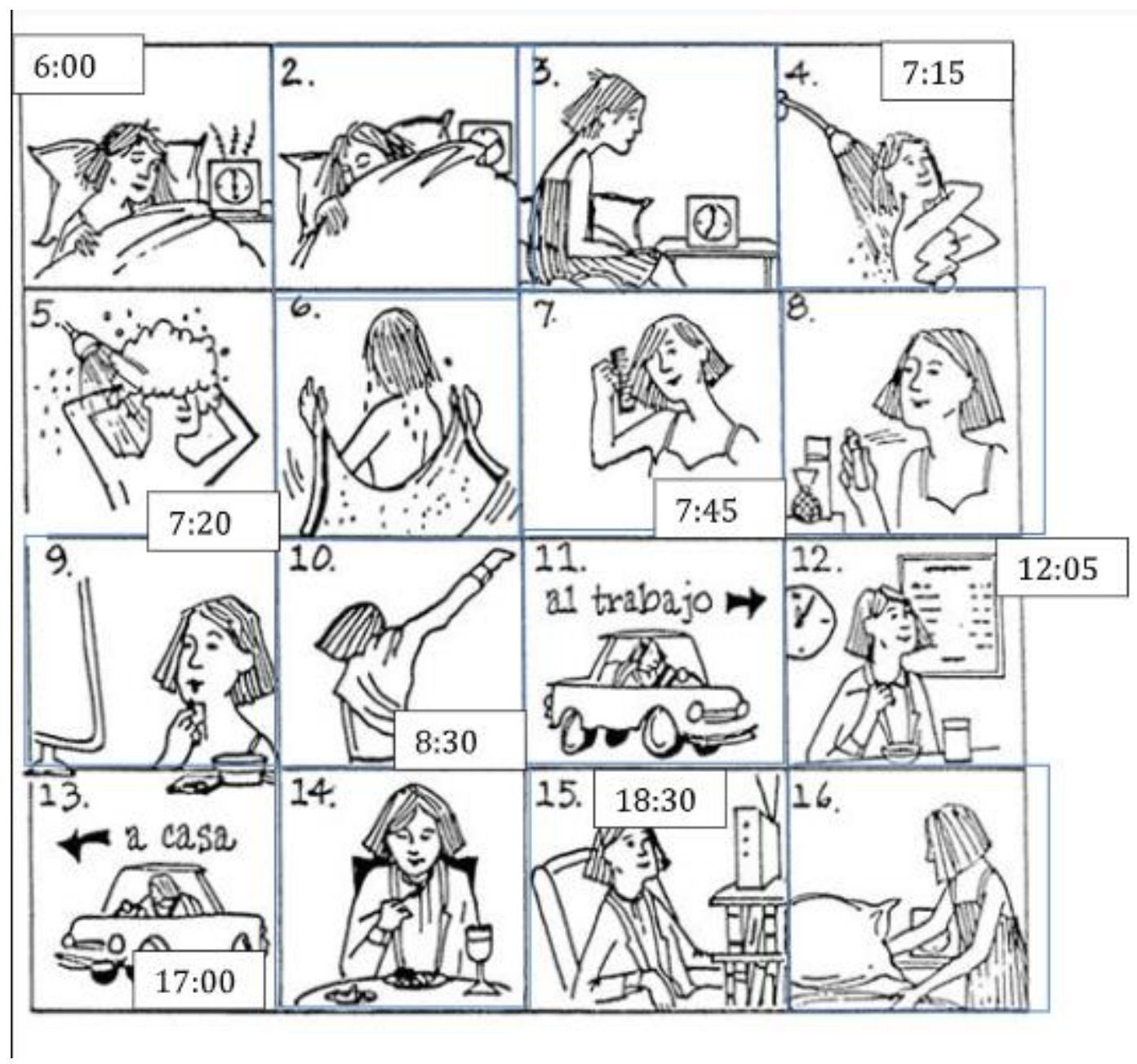

THIS TASK IS COMPLETED ONLY IN SPANISH. ASK YOUR PARTNER QUESTIONS IN SPANISH IF YOU DO NOT KNOW THE WORD.

¿Cómo se dice wake up? Se dice despertarse ¿Qué significa despertarse? Significa wake up.

Repite por favor

¿Qué hace Juana en numero 4? 